\title{
Deformation of Elastomeric Networks: Relation between Molecular Level Deformation and Classical Statistical Mechanics Models of Rubber Elasticity
}

\author{
J. S. Bergström* and M. C. Boyce \\ Department of Mechanical Engineering, \\ Massachusetts Institute of Technology, Cambridge \\ *Email address: jorgen@PolymerFEM.com \\ The final version of this draft working paper is published in: \\ Macromoleclues, Vol. 32, pp. 3795-3808, 2001.
}

\begin{abstract}
In this work a specialized molecular simulation code has been used to provide details of the underlying micromechanisms governing the observed macroscopic behavior of elastomeric materials. In the simulations the polymer microstructure was modeled as a collection of unified atoms interacting by two-body potentials of bonded and non-bonded type. Representative Volume Elements (RVEs) containing a network of 200 molecular chains of 100 bond lengths are constructed. The evolution of the RVEs with uniaxial deformation was studied with molecular dynamics techniques. The simulations enable observation of structural features with deformation including bond lengths and angles as well as chain lengths and angles. The simulations also enable calculation of the macroscopic stress-strain behavior and its decomposition into bonded and non-bonded contributions. The distribution in initial end-to-end chain lengths is consistent with Gaussian statistics treatments of rubber elasticity. It is shown that application of an axial strain of $+/-0.7$ (a logarithmic strain measure is used) only causes a change in the average bond angle of $-/+5$ degrees indicating the freedom of bonds to sample space at these low-moderate deformations; the same strain causes the average chain angle to change by $-/+20$ degrees. Randomly selected individual chains are monitored during deformation; their individual chain lengths and angles are found to evolve in an essentially affine manner consistent with Gaussian statistics treatments of rubber elasticity. The average chain length and angle are found to evolve in a manner consistent with the eight-chain network model of elasticity. Energy quantities are found to remain constant during deformation consistent with the nature of rubber elasticity begin entropic in origin. The stress-strain response is found to have important bonded and non-bonded contributions. The bonded contributions arise from the rotations of the bonds toward the maximum principal stretch axis(es) in tensile(compressive) loading.
\end{abstract}




\section{Introduction}

The macromolecular network structure of elastomeric materials provides the ability of these materials to undergo large strain, nonlinear elastic deformations. The underlying structure is essentially one of long, randomly oriented molecular chains in a network arrangement due to sparse cross-linking between the long chain molecules; furthermore, the intermolecular interactions are weak. The nature of this structure results in a stress-strain behavior that is governed by changes in configurational entropy as the randomly-oriented molecular network becomes preferentially-oriented with stretching. The essence of this behavior has been well-modeled by statistical mechanics treatments of rubber elasticity (for example, see Treloar [1975] for a review). In this work, we first provide a brief review of a few selected models of rubber elastic deformation for purpose of later comparison and we then present a molecular dynamics (MD) simulation of an elastomeric network and its deformation. The MD simulation provides detailed insight into the initial molecular network structure as well as the evolution of the network with deformation and the resulting stress-strain behavior. The relationships among the structural evolution observed in the MD simulations, the statistical mechanics models, and the continuum mechanics models of rubber elasticity are also discussed.

\section{Statistical Mechanics Constitutive Models}

An excellent review of the development of statistical mechanics treatments of rubber elasticity is given

in Treloar [1975]; therefore, only basic aspects are provided here for the purpose of later comparisons. The statistical mechanics approach begins by assuming a structure of randomly-oriented long molecular chains. In the Gaussian treatment [Wall, 1942, Treloar, 1943] the distribution of the end-to-end length, $l$, of a chain is given by $P(l)$ :

$$
P(l)=4 \pi\left(\frac{3}{2 \pi n b^{2}}\right)^{3 / 2} l^{2} \exp \left(-\frac{3 l^{2}}{2 n b^{2}}\right)
$$

where $n$ is the number of links in the chain and $b$ is the length of each link. The average initial chain length, $l_{0}$, is given by the root-mean-square value of $l$ :

$$
l_{0}=\left\langle l^{2}\right\rangle^{1 / 2}=b \sqrt{n} .
$$

When deformation is applied, the chain structure stretches. If one considers the deformation of an assembly of $N$ chains per unit reference volume by a principal stretch state $\left(\lambda_{1}, \lambda_{2}, \lambda_{3}\right)$ and the deformation is such that the chain length $l$ does not approach its fully extended length $l_{\max }=n b$, then the elastic strain energy density function, $W$, can be derived from the change in configurational entropy:

$$
W=\frac{1}{2} N k_{B} \theta\left(\lambda_{1}^{2}+\lambda_{2}^{2}+\lambda_{3}^{2}-3\right)
$$

where $k_{B}$ is Boltzmann's constant and $\theta$ is absolute temperature. The stress-stretch relationship is then found by differentiating the strain energy density function.

The derivation of Equation (3) relies on $l \ll n b$. At deformations where $l$ begins to approach the fully extended length, the non-Gaussian nature of the chain stretch must be taken into account. Kuhn and Grün [1942] accounted for the finite extensibility of chain stretch using Langevin chain statistics. 
To incorporate these more accurate chain statistics into a constitutive framework, it is necessary to have a model that relates the chain stretch to the applied deformation; this is accomplished by assuming a representative network structure. Three network models are shown in Figure 1. The unit cell used

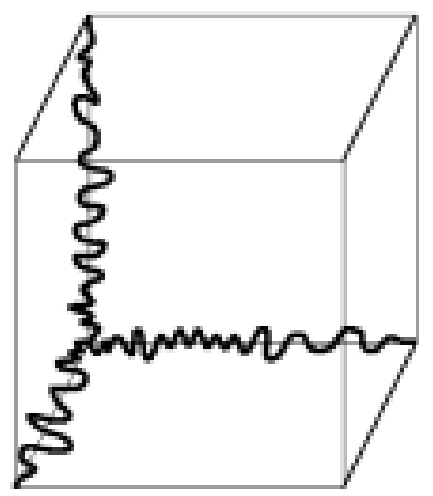

(a)

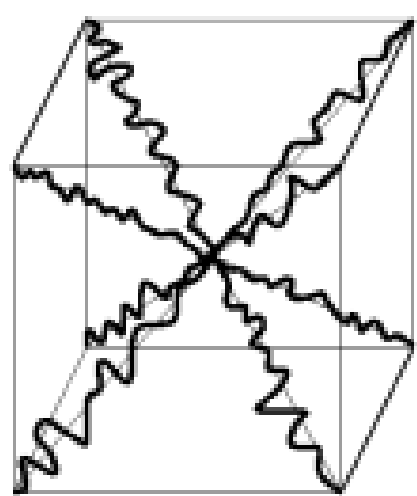

(b)

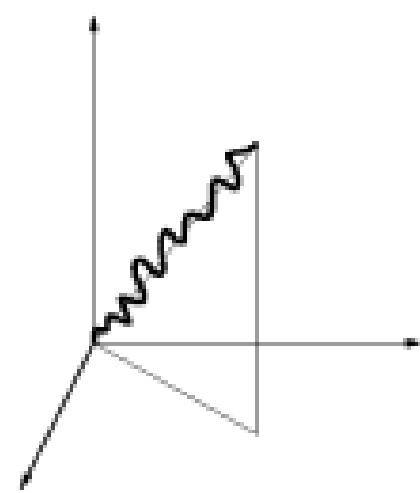

(c)

Figure 1: Different chain topologies: (a) 3-chain; (b) 8-chain; (c) full network.

in each of these models is taken to deform in principal stretch space; the models differ in how the deformation of the chains is related to the deformation of the unit cell. In the "3-chain" model [Wang and Guth, 1952], the chains are located along the axes of the cell; in the "8-chain" model [Arruda and Boyce, 1993] the chains are located along the diagonals of the unit cell; and in the full-network or total assembly of chains model [Treloar, 1954, Wu and van der Giessen, 1993], the chains are assumed to be randomly distributed in space and to deform in an affine manner. Of these models, the 8-chain representation appears to give the best predictions when compared to experimental data [Arruda and Boyce, 1993, Wu and van der Giessen, 1993]. The strain energy density for the Arruda-Boyce model (assuming incompressibility) is given by:

$$
W=N k \theta \sqrt{n}\left[\beta \lambda_{\text {chain }}-\sqrt{n} \ln \left(\frac{\sinh \beta}{\beta}\right)\right],
$$

where

$$
\begin{aligned}
\lambda_{\text {chain }} & =\sqrt{\frac{1}{3}\left(\lambda_{1}^{2}+\lambda_{2}^{2}+\lambda_{3}^{2}\right)}, \\
\beta & =\mathcal{L}^{-1}\left(\frac{\lambda_{\text {chain }}}{\sqrt{n}}\right),
\end{aligned}
$$

where $\lambda_{\text {chain }}$ is the stretch in each chain in the network and $\mathcal{L}^{-1}(x)$ is the inverse Langevin function that can be estimated from:

$$
\mathcal{L}^{-1}(x)= \begin{cases}1.31446 \tan (1.58986 x)+0.91209 x, & \text { if }|x|<0.84136, \\ 1 /(\operatorname{sign}(x)-x), & \text { if } 0.84136 \leq|x|<1\end{cases}
$$

For small deformations, the 8-chain model reduces to the Gaussian model as do the 3-chain and fullnetwork models.

The success of the statistical mechanics models of rubber elasticity can now be explored in detail us- 
ing molecular level modeling. Below, simulation of the structure and the deformation of an elastomeric network are presented; results are compared to the models presented above where possible.

\section{Molecular Model Description}

One of the most important choices when developing a molecular simulation code is the decision of what potential energies to use. To a large extent the physical behavior of a material is governed by how the atoms interact with each other, i.e.by the potential energies of the interactions. When studying the mechanical properties of a polymeric material, however, the behavior is mainly governed by the topological features of the microstructure such as network structure and entanglements, and using a coarse graining procedure can therefore be a successful approach. To optimize the simulation code for speed it is desirable to use potential energies that are as simple as possible, while still keeping the necessary chain-like features of the polymer microstructure. In this work the focus is on crosslinked polymeric systems above the glass transition temperature, the behavior of glassy polymers using similar techniques has recently been studied by Chui and Boyce [1999]. Above the glass transition temperature the thermal energy is so high that it is possible to neglect the energy associated with bond angle rotations and torsions, and it is therefore sufficient to use simple bonded and non-bonded potentials when representing the microstructure. The bonded potential is here taken as a linear harmonic spring:

$$
U_{B}^{t o t}=\sum_{\alpha \in b} \frac{c_{B}}{2}\left[r_{\alpha}-b\right]^{2}
$$

where the sum is over all bonds $\alpha, c_{B}$ is the spring stiffness, $b$ the bond length at zero force, and $r_{\alpha}$ the length of bond $\alpha$. The interchain potential is represented with a shifted and truncated Lennard-Jones potential $^{1}$ :

$$
U_{L J}^{t o t}=\sum_{\alpha \in n b} U_{L J}\left(r_{\alpha}\right)
$$

where the sum is over all pairs of atoms $\alpha$, unless the pair of atoms are nearest chain neighbors, and where

$$
U_{L J}\left(r_{\alpha}\right)= \begin{cases}4 \epsilon_{L J}\left[\left(\frac{\Lambda}{r_{\alpha}}\right)^{12}-\left(\frac{\Lambda}{r_{\alpha}}\right)^{6}-\left(\frac{1}{r_{c}}\right)^{12}+\left(\frac{1}{r_{c}}\right)^{6}\right], & \text { if } r_{\alpha}<r_{c} \Lambda, \\ 0 \quad & \text { otherwise. }\end{cases}
$$

In Equation (10), $\epsilon_{L J}$ is the Lennard-Jones potential strength, $r_{c}$ is the cut-off distance, $\Lambda$ is the excluded volume parameter, and $r_{c} \Lambda$ the interaction distance.

In molecular simulations most of the time is spent calculating the potential energies or gradients of the potential energies. Direct computation of these terms scale as $M^{2}$, where $M$ is the number of atoms. In order to minimize the computational effort, a cutoff distance, $r_{c}$, is implemented such that all interactions at $r>r_{c}$ are ignored. Different techniques can be used to monitor which atoms are within the cutoff distance. The two techniques used here are the Verlet neighbor list [Verlet, 1967] and the link-cell method [Hockney et al., 1974].

In molecular simulations it is often convenient to express quantities such as temperature, density, pressure, etc., in reduced units. This means that a convenient set of base variables is chosen and then

\footnotetext{
${ }^{1}$ Note that although this potential is continuous, its gradient is not continuous. This, however, does not cause any problems in the large scale simulations used in this work in which long terms statistical averages are the main interest.
} 
all other quantities are normalized with respect to these base variables. A natural choice for base variables in this case are: $m$ (atom mass), $\theta$ (temperature), $b$ (bond length), and $k_{B}$ (Boltzmann's constant). Normalized base units can then be written:

$$
\begin{aligned}
& {[\text { energy }] } \triangleq k_{B} \theta, \\
&\text { force }] \triangleq \frac{k_{B} \theta}{b}, \\
& {[\text { time }] \triangleq \sqrt{\frac{m b^{2}}{k_{B} \theta}}, } \\
& {[\text { velocity }] \triangleq \sqrt{\frac{k_{B} \theta}{m},} } \\
& {[\text { acceleration }] } \triangleq \frac{k_{B} \theta}{m b} .
\end{aligned}
$$

Reduced variables, denoted with a tilde, can then be expressed in terms of these reduced base units, e.g. $c_{b}=\tilde{c}_{b}\left(k_{b} \theta / b^{2}\right)$. The most important reason for introducing reduced units is that infinitely many combinations of the input variables correspond to the same state in terms of reduced units. Another practical reason for using reduced units is to make the magnitude of almost all quantities to be $\mathcal{O}(1)$, and hence reduce the problem of truncation errors when implemented on a finite precision computer.

The crosslinking procedure used in this work is similar to that used by Duering [1994]. First, a melt of linear monodispersed chains is generated and reactive tetrafunctional groups added to all chain ends. The network structure is then generated by running an initial Monte Carlo based crosslinking step during which chain ends that are within a predefined distance from each other are permanently attached. To further speed up the crosslinking step an artificial potential energy is introduced between all chain ends

$$
U_{C L}^{t o t}=\sum_{\alpha \in c e} U_{C L}\left(r_{\alpha}\right)
$$

where the sum is over all pairs of chain ends and where

$$
U_{C L}\left(r_{\alpha}\right)=\frac{-K_{C L} \phi}{r_{\alpha}}
$$

The constant $K_{C L}$ determines the strength of the crosslinking potential, and $\phi$ is 1 if the sum of the current functionalities of the two chain ends is less than or equal to 4 and $\phi$ is -1 otherwise. In some simulations both the reduced density and the temperature was increased to further facilitate the crosslinking procedure.

Calculation of stresses can be accomplished with the virial stress theorem from statistical mechanics (for example, Gao and Weiner [1987]). In this case with a system of atoms interacting with two-body potentials the Cauchy stress is given by

$$
V T_{i j}=-M k_{B} \theta \delta_{i j}+\sum_{\alpha \in b}\left\langle\frac{r_{\alpha i} r_{\alpha j}}{r_{\alpha}} \frac{\partial U_{B}^{t o t}}{\partial r_{\alpha}}\right\rangle+\sum_{\alpha \in n b}\left\langle\frac{r_{\alpha i} r_{\alpha j}}{r_{\alpha}} \frac{\partial U_{L J}^{t o t}}{\partial r_{\alpha}}\right\rangle
$$

where $V$ is the volume of the RVE, $M$ is the number of atoms in the RVE, $r_{\alpha}$ is the distance between the pair of atoms $\alpha, r_{\alpha i}$ is the $i$ th component of $r_{\alpha}$, and $\langle\cdot\rangle$ represent ensemble or time average. Using Equation (18) the stresses in the three axial directions can be calculated. In $N V \theta$ simulations (often 
called NVT simulations, but $T$ is here used to symbolize Cauchy stress), the volume is held constant which can give rise to a pressure. Therefore, in incompressible uniaxial deformation the stresses also contain a pressure contribution, i.e.this hydrostatic stress needs to be taken into account giving the axial stress as $\sigma=T_{x x}-\frac{1}{2}\left(T_{y y}+T_{z z}\right)$.

Once the initial network structure and the potential energies have been specified the prescribed boundary displacements can be applied. To follow the structure evolution of the polymeric system a number of different techniques can be used; in this work a molecular dynamics algorithm has been employed. The details of this approach are described in the next subsection.

\subsection{Molecular Dynamics Algorithm}

The MD simulations consider a canonical ensemble $(N, V, \theta)$. The $(N, V, \theta)$ ensemble necessitates a modification of the equations of motion with an additional degree of freedom representing a kinetic mass [Nosé, 1984, Hoover, 1985]:

$$
m \frac{d^{2} \mathbf{r}_{i}}{d t^{2}}=-\nabla U-\eta \mathbf{v}_{i}, \quad i \in[1, N]
$$

where $m$ is the mass of unified atom $i$ and $\mathbf{r}_{i}$ its position, $U$ the total potential energy, and where the evolution of the parameter $\eta$ is given by

$$
\dot{\eta}=\frac{1}{Q}\left[\sum_{i} v_{i}^{2}-f\right]
$$

where $f$ is the number of degrees of freedom of the system, and $Q$ a kinetic mass.

The numerical integration of Equations (19) and (20) can be performed in many different ways, one common method is the Velocity Verlet algorithm which can be written [Allen and Tildesley, 1987]:

$$
\begin{aligned}
& \mathbf{r}(t+\Delta t)=\mathbf{r}(t)+\Delta t \mathbf{v}(t)+\frac{\Delta t^{2}}{2} \mathbf{a}(t) \\
& \mathbf{v}(t+\Delta t)=\mathbf{v}(t)+\frac{\Delta t}{2}[\mathbf{a}(t)+\mathbf{a}(t+\Delta t)]
\end{aligned}
$$

To use this algorithm in a Nosé-Hoover based MD code it is necessary to first rewrite (19) as

$$
m \mathbf{a}(t+\Delta t)=-\nabla U(\mathbf{r}(t+\Delta t))-\eta(t+\Delta t) \mathbf{v}(t+\Delta t)
$$

Inserting this equation into (22) results in

$$
\mathbf{v}(t+\Delta t)=\mathbf{v}(t)+\frac{\Delta t}{2}\left\{\frac{-1}{m} \nabla U(\mathbf{r}(t+\Delta t))-\frac{\eta(t+\Delta t)}{m} \mathbf{v}(t+\Delta t)+\mathbf{a}(t)\right\} .
$$

Solving for $\mathbf{v}(t+\Delta t)$ gives

$$
\mathbf{v}(t+\Delta t)=\left[1+\frac{\eta(t+\Delta t) \Delta t}{2 m}\right]^{-1}\left\{\mathbf{v}(t)-\frac{\Delta t}{2 m} \nabla U(\mathbf{r}(t+\Delta t))+\frac{\Delta t \mathbf{a}(t)}{2}\right\} .
$$

The MD procedure then simply becomes: 
1. start with a given initial configuration: $\mathbf{r}_{i}(t), \mathbf{v}_{i}(t), \mathbf{a}_{i}(t)$

2. calculate $\mathbf{r}_{i}(t+\Delta t)$ with $(21)$

3. calculate $\eta(t+\Delta t)=\eta(t)+\Delta t \dot{\eta}(t)+\frac{\Delta t^{2}}{2} \ddot{\eta}(t)$

4. calculate $\mathbf{v}_{i}(t+\Delta t)$ with $(25)$

5. calculate $\dot{\eta}(t+\Delta t)$ with $(20)$

6. calculate $\mathbf{a}_{i}(t+\Delta t)$ with (23)

7. calculate $\ddot{\eta}(t+\Delta t)=\frac{2}{Q} \sum \mathbf{v}(t+\Delta t) \mathbf{a}(t+\Delta t)$

8. save data and goto 2

To start the MD simulation both the initial velocities and the initial accelerations of all atoms need to be specified. The accelerations can all be taken to be zero, but to get the right kinetic energy and therefore also temperature it is sufficient to choose the velocity components $v_{i x}$ from the Gaussian distribution

$$
\rho\left(v_{i x}\right)=\sqrt{\frac{m}{2 \pi k_{B} \theta}} \exp \left[\frac{-m v_{i x}^{2}}{2 k_{B} \theta}\right],
$$

where $\rho\left(v_{i x}\right)$ is the probability density for velocity component $v_{i x}$.

\section{Initial Structure}

A well equilibrated initial structure is constructed by first randomly generating a melt of all chains. The melt was then allowed to relax for an extended amount of time using a Monte Carlo (MC) method to make the system reach a local energy minimum. The system was then crosslinked using the MC-based crosslinking algorithm described in Section 3.

The size of the unit cell used in all simulations was rather small containing only $2 \cdot 10^{4}$ unified atoms configured into 200 chains, see the schematic representation in Figure 2. In this figure the diameter of the atoms has been reduced to make it easier to distinguish the different regions of the system. The input parameters used in the simulations are summarized in Table 1. Results from this type of

\begin{tabular}{cccccc}
\hline \hline density & temperature & cut-off & excluded volume & kinetic & spring \\
& & distance & parameter & mass & stiffness \\
$\tilde{\rho}$ & $\tilde{\theta}$ & $\tilde{r}_{c}$ & $\Lambda$ & $\tilde{Q}$ & $\tilde{c}_{B}$ \\
\hline 0.85 & 2 & 1.4 & 1 & 500 & 800 \\
\hline \hline
\end{tabular}

Table 1: Input parameters used in the molecular simulations.

simulation are presented in Figure 3 illustrating that the number of free chains is close to zero and that the number of dangling chain-ends is quite small. The radial distribution function in the undeformed state, plotted in Figure 4, demonstrates a peak at the first nearest neighbor distance. At larger distance the spatial correlation becomes more random causing $g(r) \rightarrow 1$. To examine the initial structure of the simulated system, Figure 5 shows the distribution of bond lengths in the undeformed state illustrating 


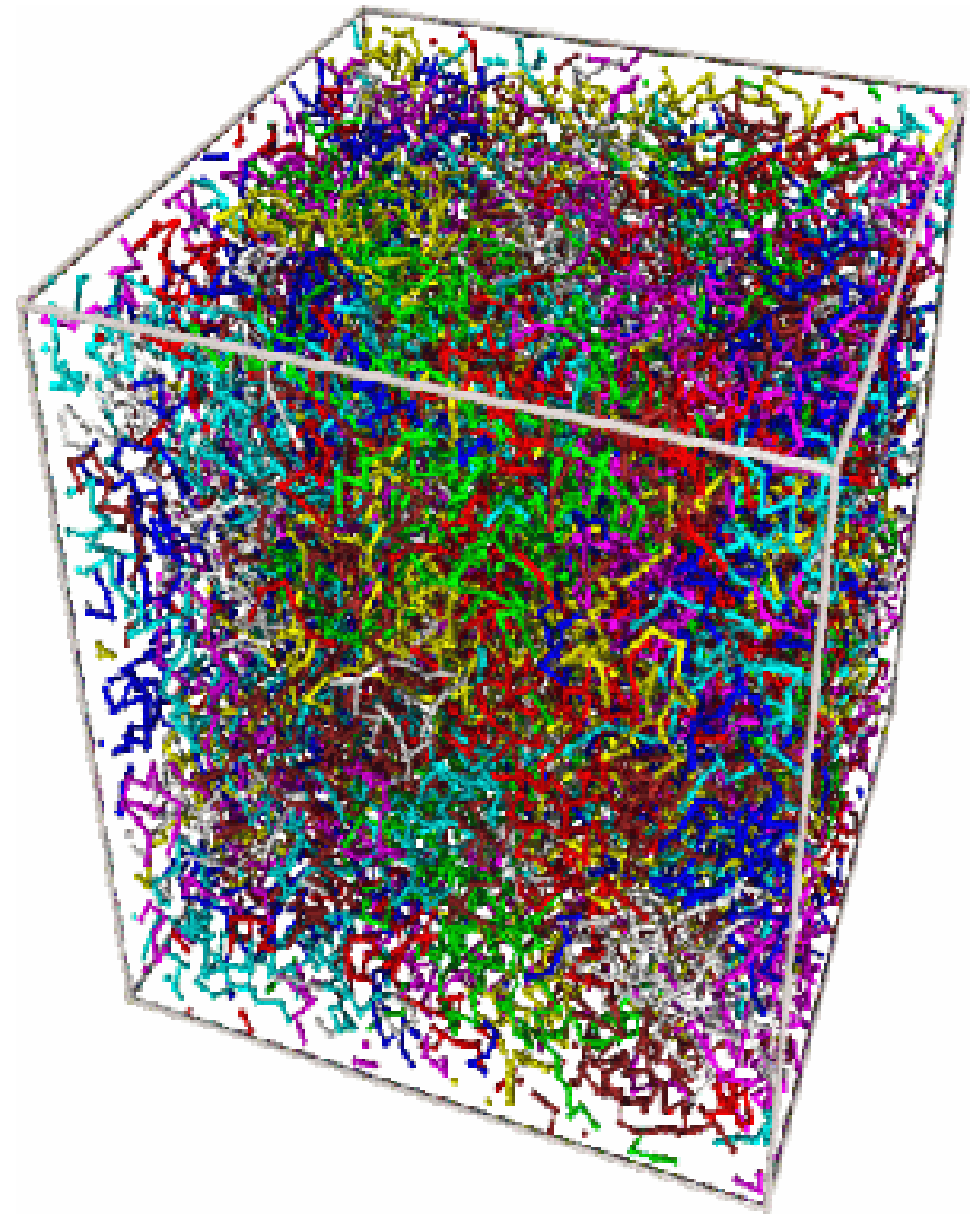

Figure 2: Distribution of atoms in the RVE in the undeformed state, $M=20000$. 


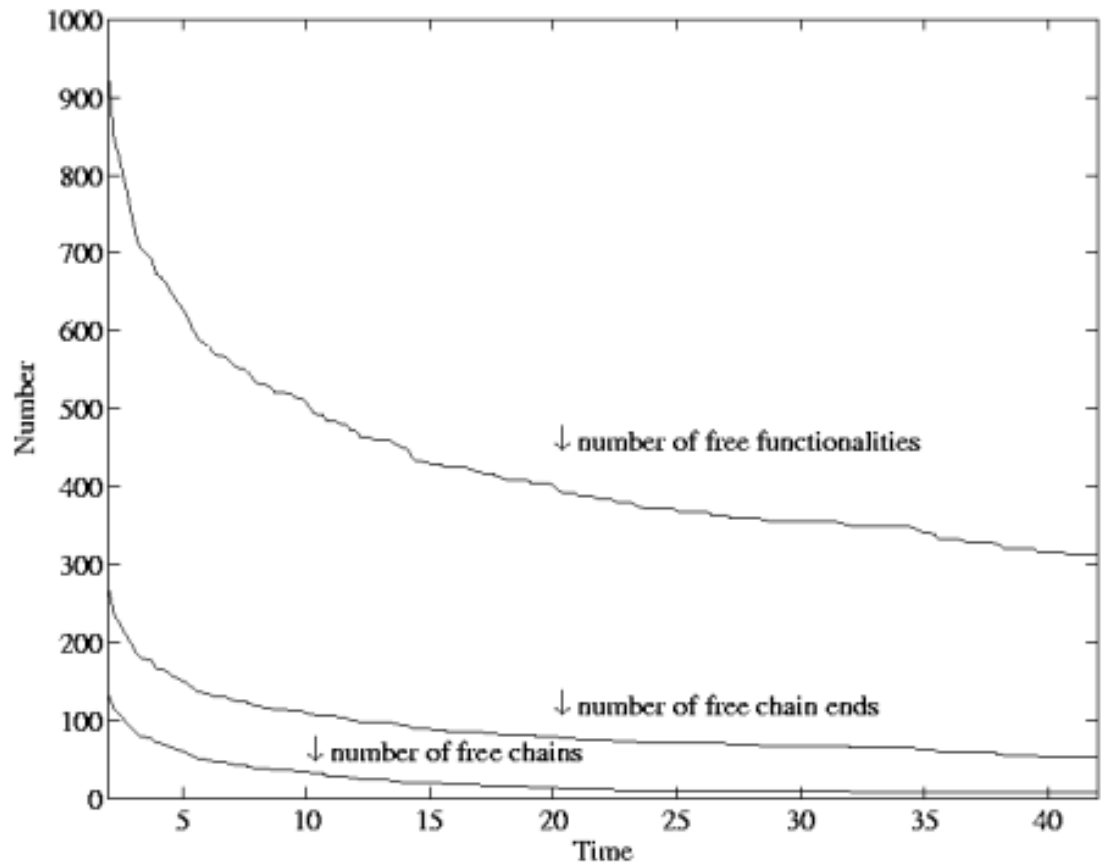

Figure 3: Crosslinking of the RVE as a function of simulation time (MC, $2 \cdot 10^{4}$ atoms, 200 chains).

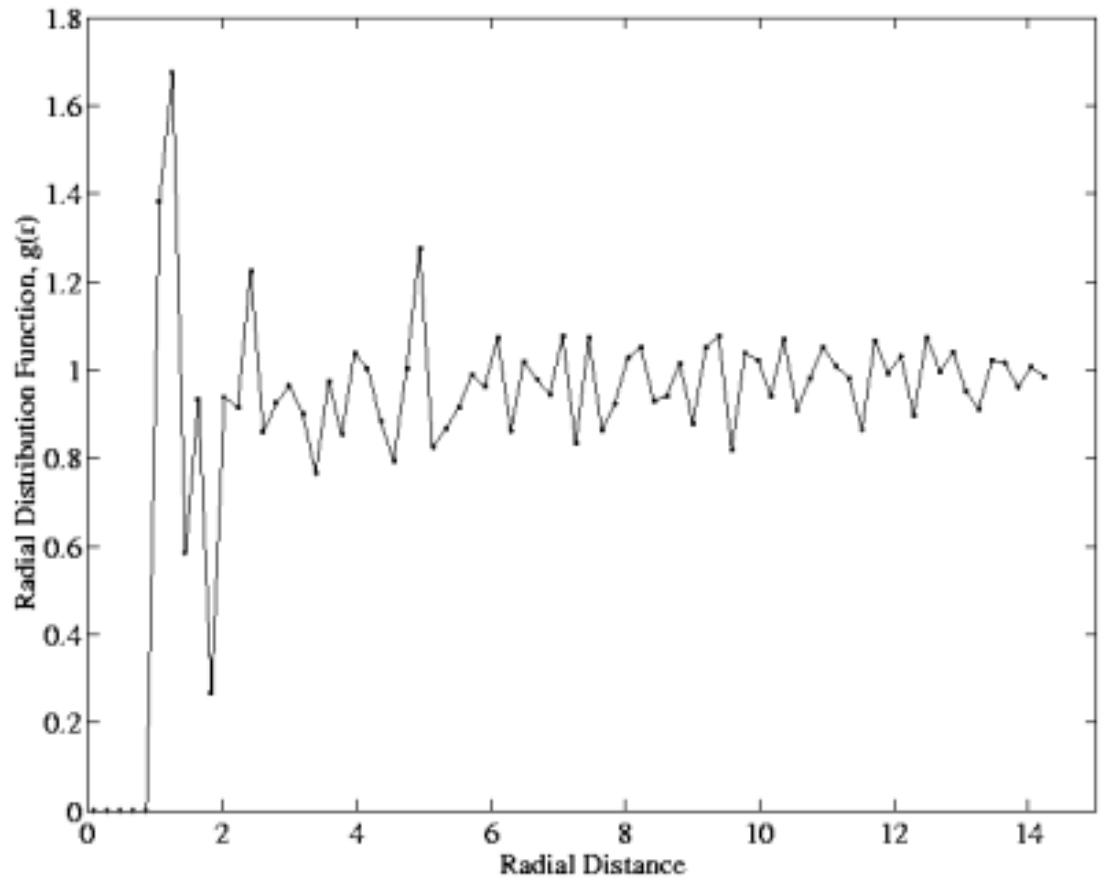

Figure 4: Radial distribution function of the simulated system in the undeformed state. 
that virtually all bonds have a length between 0.9 and 1.1. The distribution in chain lengths is shown

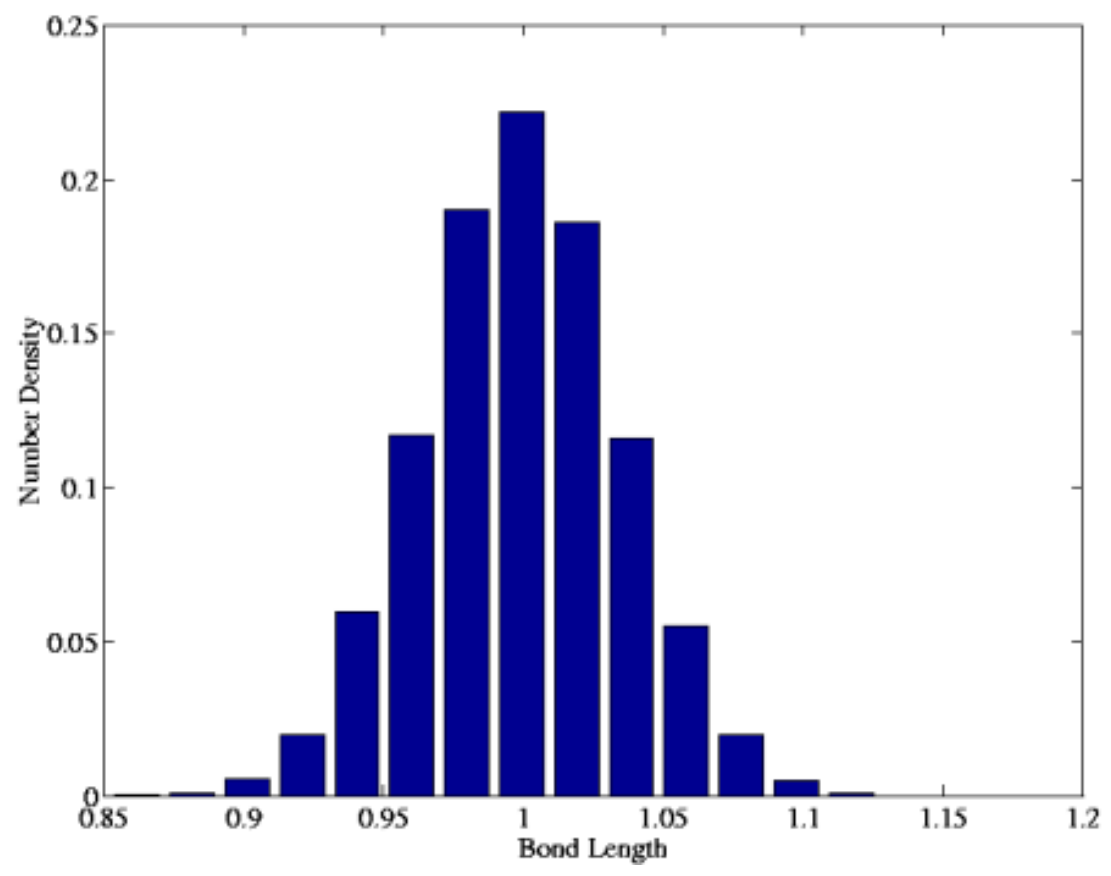

Figure 5: Bond length distribution in the undeformed state.

in Figure 6. In this case each chain contained 100 bonds each of length $b=1$, and the figure shows that the average chain length is 10.24 which is in agreement with the random walk prediction $l_{0}=b \sqrt{N}$. In the figure is also plotted the distribution function representing Gaussian chain statistics given earlier in Equation (1) illustrating that the simulated chain length distribution is in good agreement with the statistical mechanics theory. The distribution in bond angles (i.e.the angle between the bond vector and the loading direction) in the undeformed state presented in a pole figure is shown in Figure 7 demonstrating a uniform random distribution. Figure 8 shows the distribution in chain angles (i.e.the angle between the end-to-end vector of the chain and the loading direction) again demonstrating a random distribution. As a final evaluation of the initial structure, the Kuhn statistical segment length, $b_{k}=\left\langle l^{2}\right\rangle / l_{\max }$, for the system was calculated to be 1.232. This value is larger than 1 due to excluded volume interactions. 


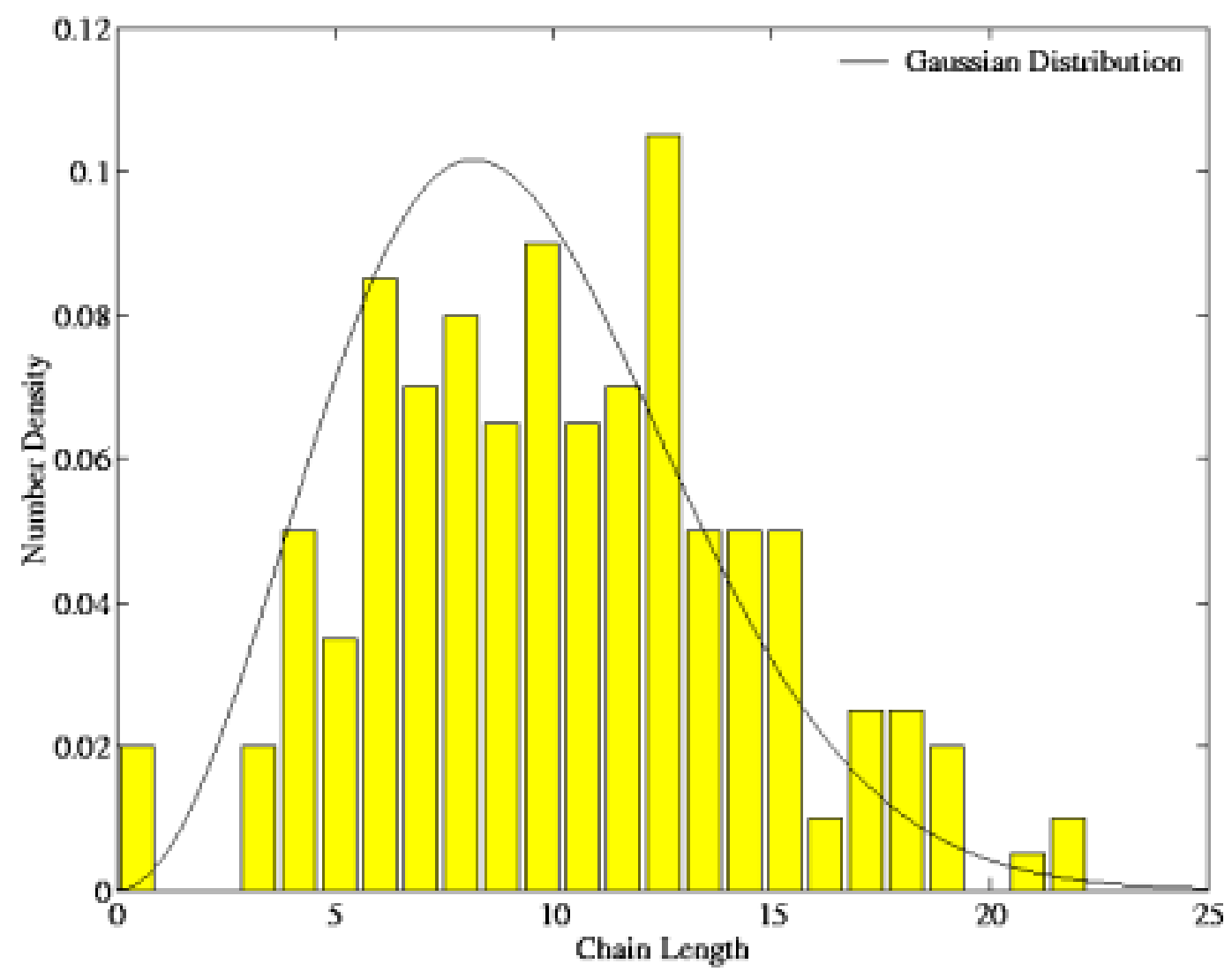

Figure 6: Chain length distribution in the undeformed state.

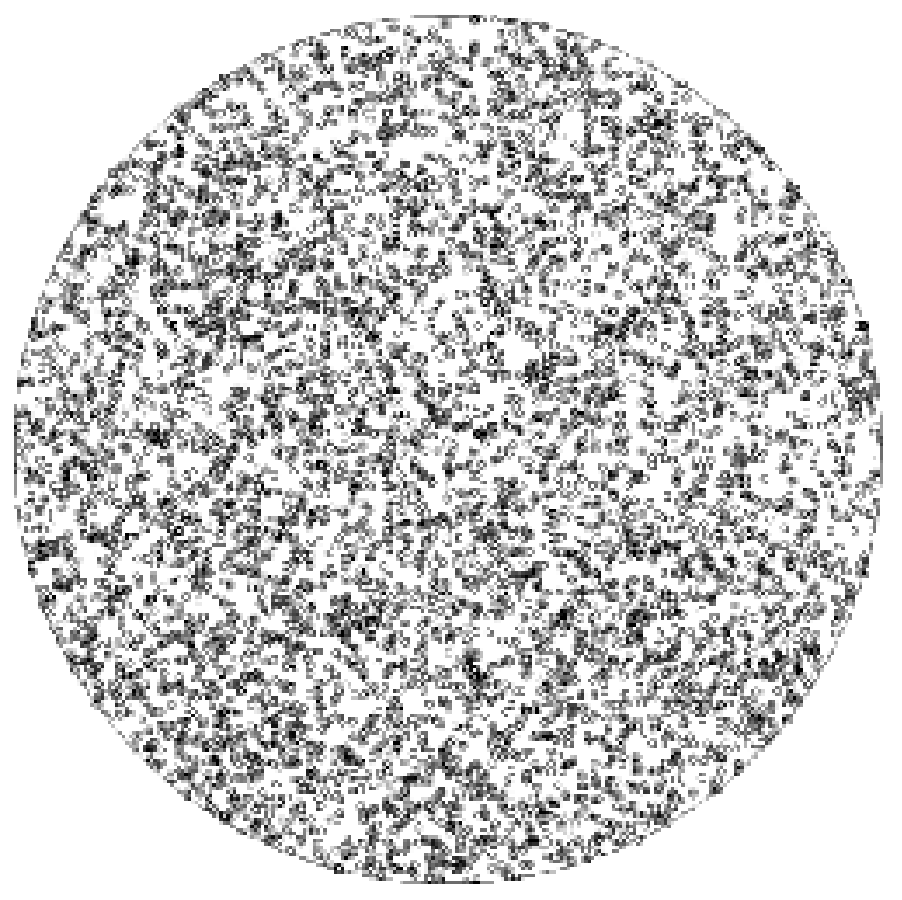

Figure 7: Bond angle pole figure of the initial state of the simulated system. 


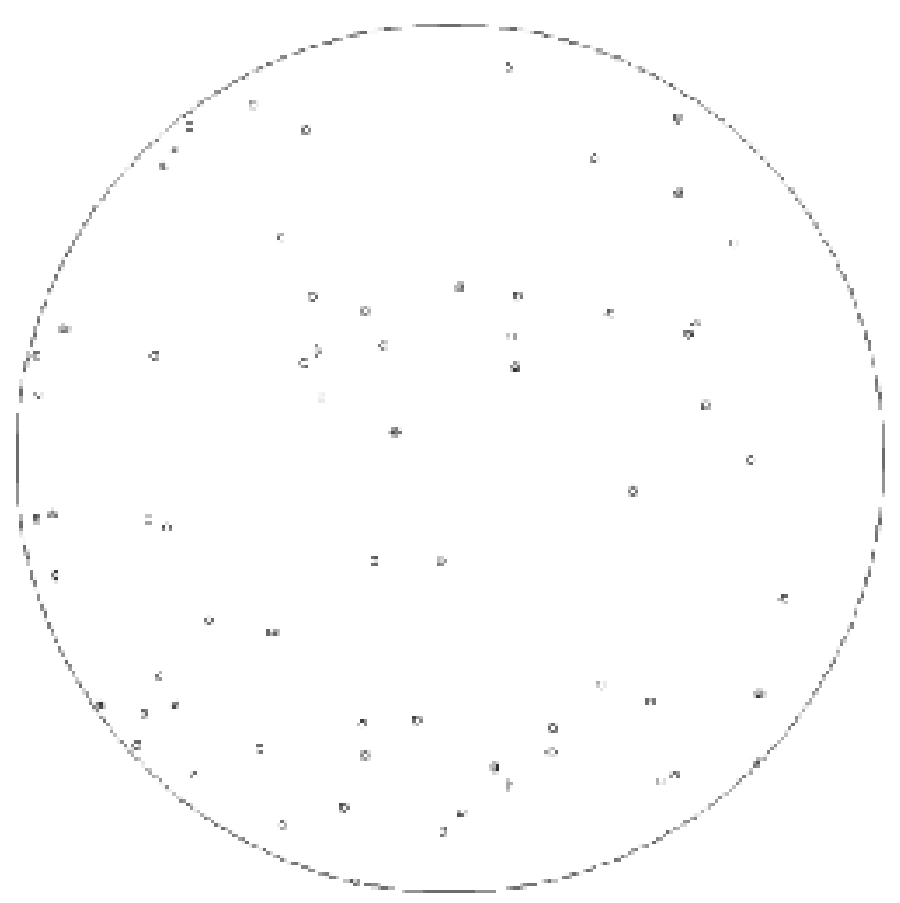

Figure 8: Chain angle pole figure of the initial state of the simulated system. 


\section{Tension Results}

A representative volume element (RVE) containing 200 chains is now deformed in tension. Results on evolution in structure with deformation and evolution in stress-strain behavior with deformation are examined.

The applied strain history used in the simulation is shown in Figure 9. In this case the RVE was

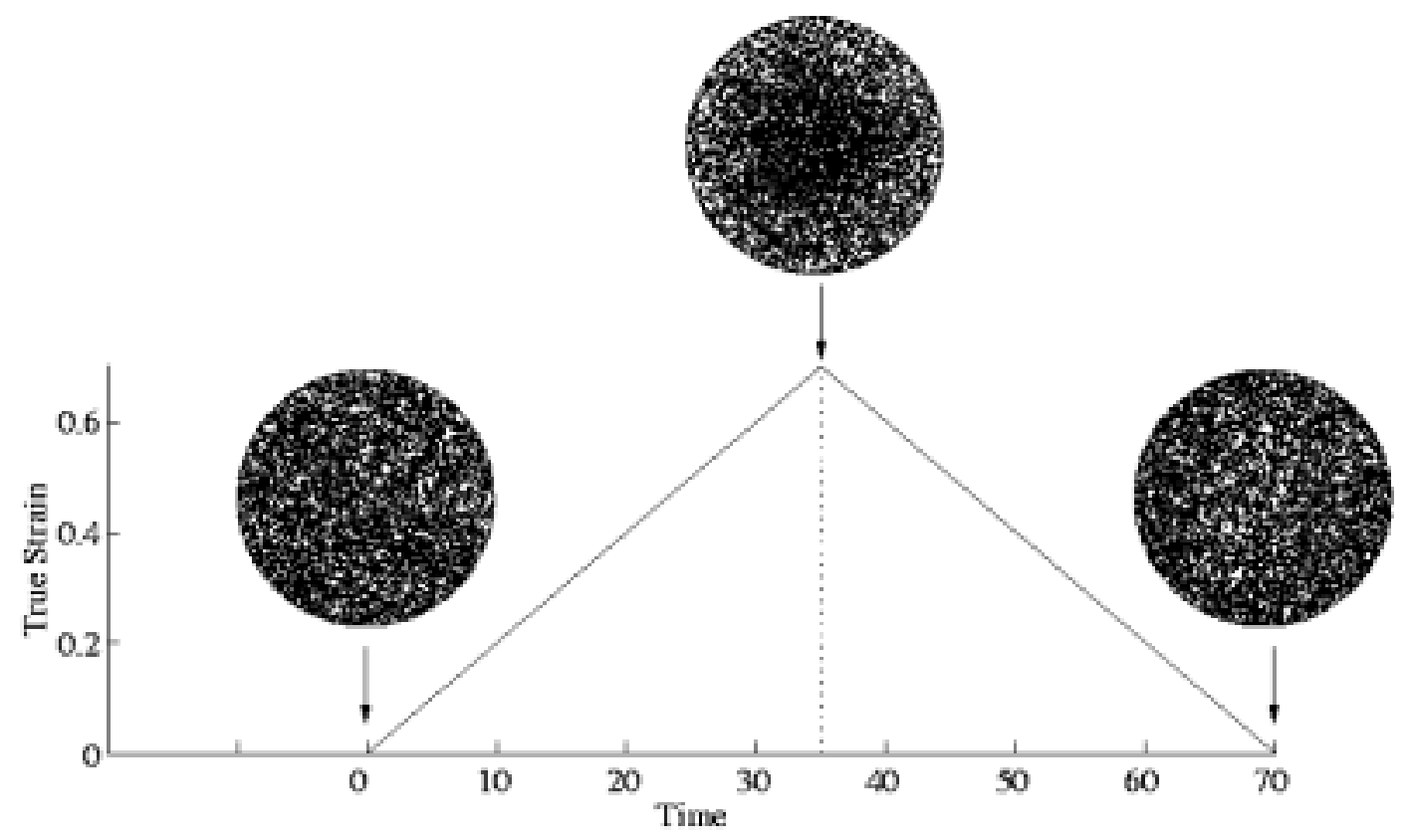

Figure 9: Applied strain history with bond angle pole figures.

uniaxially deformed in tension to a final true strain of $\varepsilon=0.7$ (a logarithmic strain measure is used), after which the applied deformation was removed with the same rate. In this and the following figures, a dotted vertical line indicates the beginning of a new loading segment. Figure 9 also depicts three bond angle pole figures illustrating that during the deformation the bond angles start to become aligned with the loading direction. The bond angle of a particular bond is here defined as the angle between the bond and the loading direction.

The maximum, average, and minimum bond lengths as a function of simulation time are shown in Figure 10 demonstrating that on average the bond lengths do not change with applied deformation. The average bond angle as a function of deformation history is shown in Figure 11. If all bonds are randomly oriented the average bond angle is given by

$$
\langle\alpha\rangle=\int_{0}^{\pi / 2} d \Theta \int_{0}^{\pi / 2} d \phi \arccos (\cos \Theta \cos \phi) \cos \Theta \approx 57.3^{\circ},
$$

which is in good agreement with the initial state of the simulated system. Figure 11 also shows that even though the RVE is stretched to a true strain of 0.7 (a tensile stretch of 2.0), the average bond angle does not change by more than about 6 degrees. After reversing the displacement, the bond angles then rotate back towards original position, but overshoot the initial angle. The effect of this on the stress-strain behavior will be shown later. 


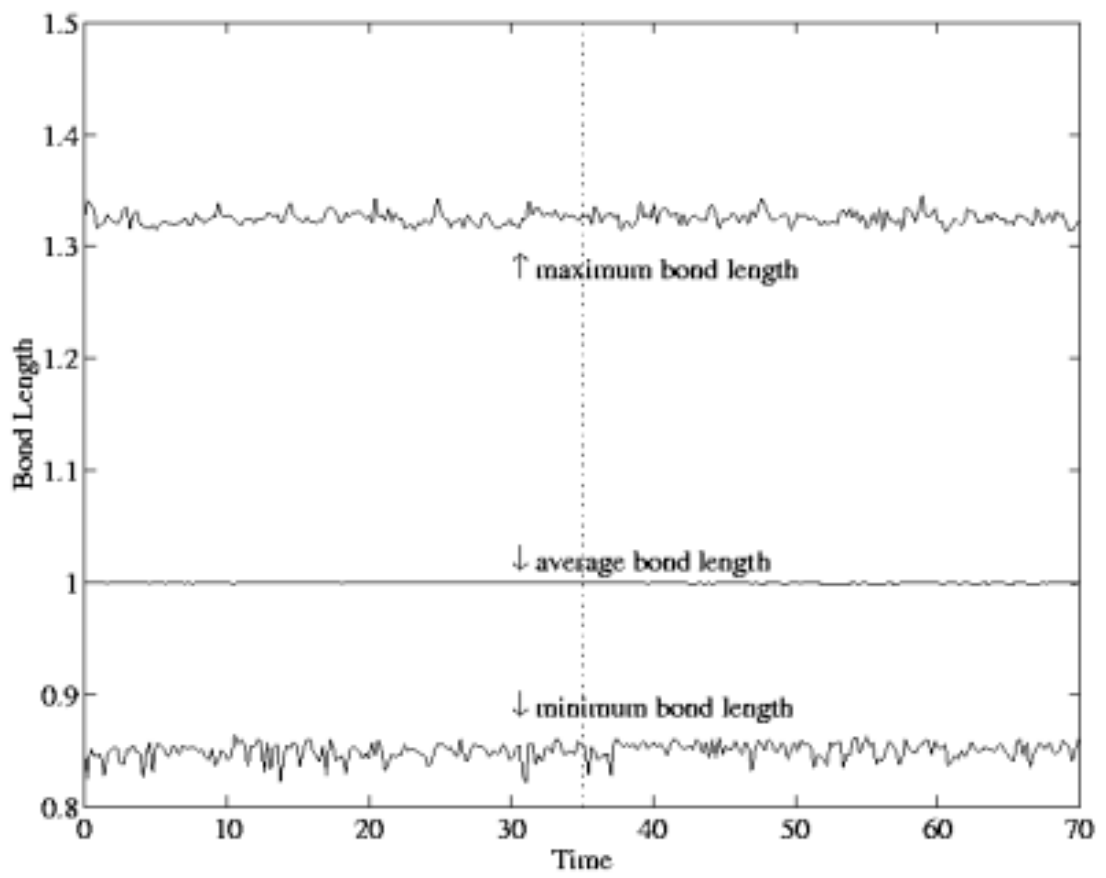

Figure 10: Maximum, average and minimum bond lengths as a function of simulation time.

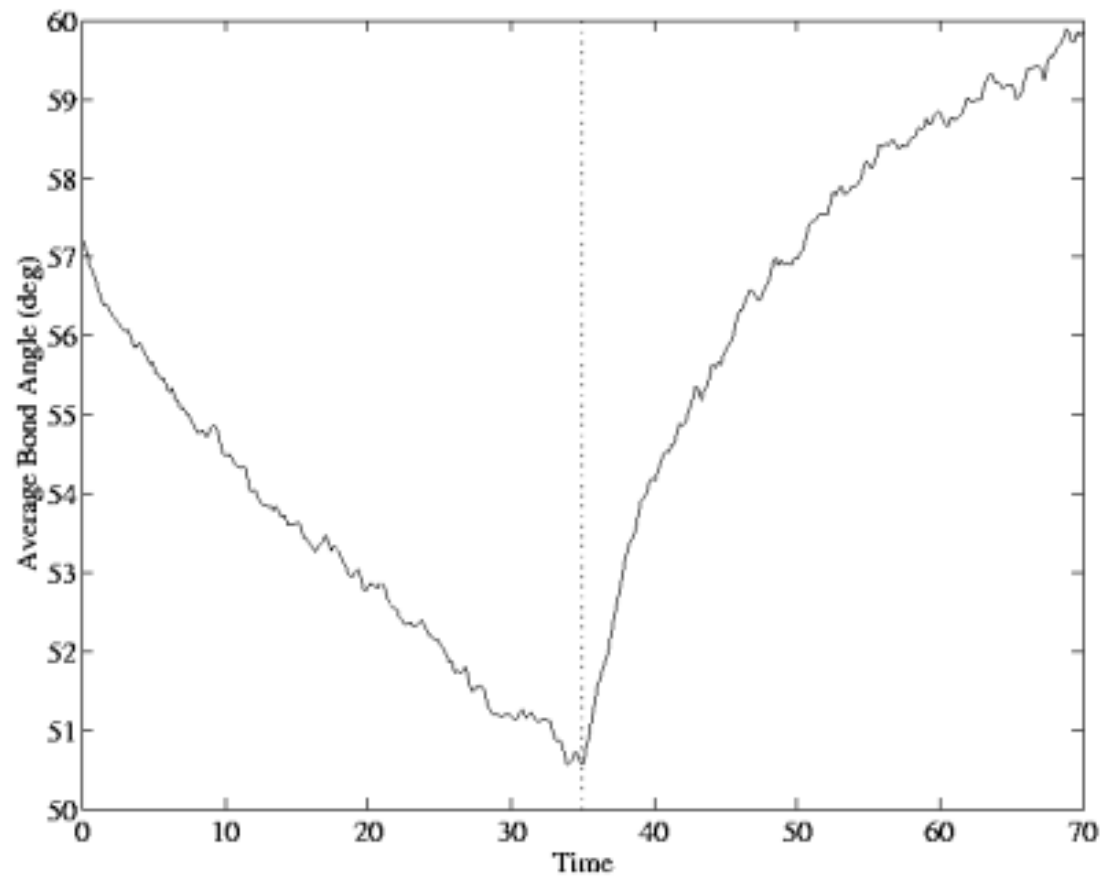

Figure 11: Average bond angle of all bonds as a function of simulation time. 
In addition to the bond angles it is also possible to follow the chain angles, as illustrated in Figures 12 and 13. In these figures is plotted the average chain angle (averaged over the 200 chains in the

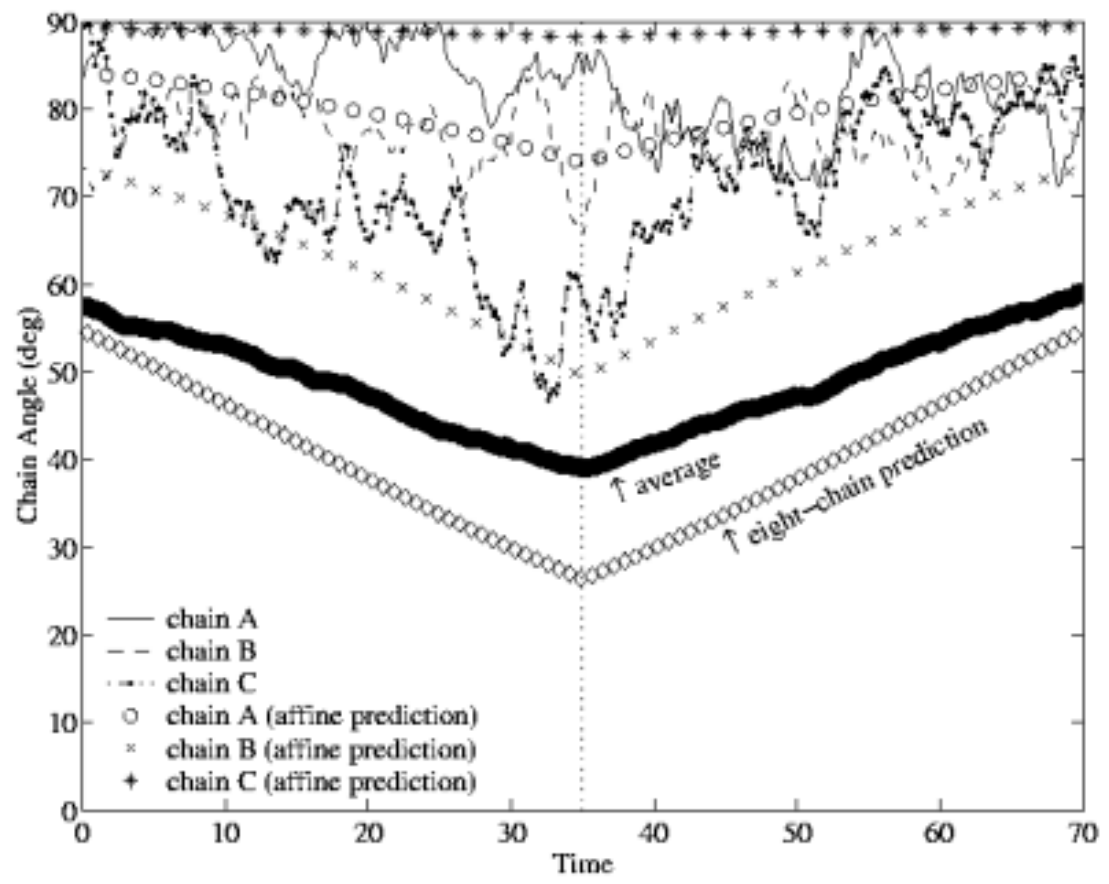

Figure 12: Chain angles as a function of simulation time.

system) as a function of deformation history. On average, the network chains are observed to rotate toward the principal stretch direction. By comparing the average chain angle with the average bond angle it is clear that they undergo similar changes, note however that the magnitude of the change of the average chain angle is about 18 degrees which is larger than the 5 degrees change in average bond angle indicating the freedom of sampling space at the bond level, consistent with Gaussian statistics assumptions (one would anticipate less freedom at large stretches). At both the initial deformation and the strain reversal point the average bond angle undergoes a more rapid change than what is observed for the average chain angle. This has direct impact on the macroscopic stress-strain behavior, as will be discussed later.

Figures 14 and 15 depict the evolutions in the end-to-end distance of six individual chains (the evolution in chain angle for these same chains was reported earlier in Figure 12 and 13). Chains which are more closely aligned with the tensile stretch axis (chains $D, E$, and $F$ ) are found to stretch as well as to rotate toward the tensile axis. It is clear that at any increment in the deformation these individual chains undergo large fluctuations in end-to-end distance and angle, but each tends toward stretching and rotating toward the principal stretch direction. Chains which are initially nearly orthogonal to the tensile axis (chains $A$ and $B$ in Figures 13 and 15) are not found to stretch and, in some cases, to even reduce the end-to-end distance.

In Figures 12 to 15 is also included the predicted affine deformation behavior of the six randomly selected chains as well as the 8-chain network model prediction of the effective network chain behavior. As discussed in Section 2, the 8-chain model possesses chains that lie along the diagonals of the cell; these chains will stretch and rotate with the deformations of the cell. For the case of uniaxial 


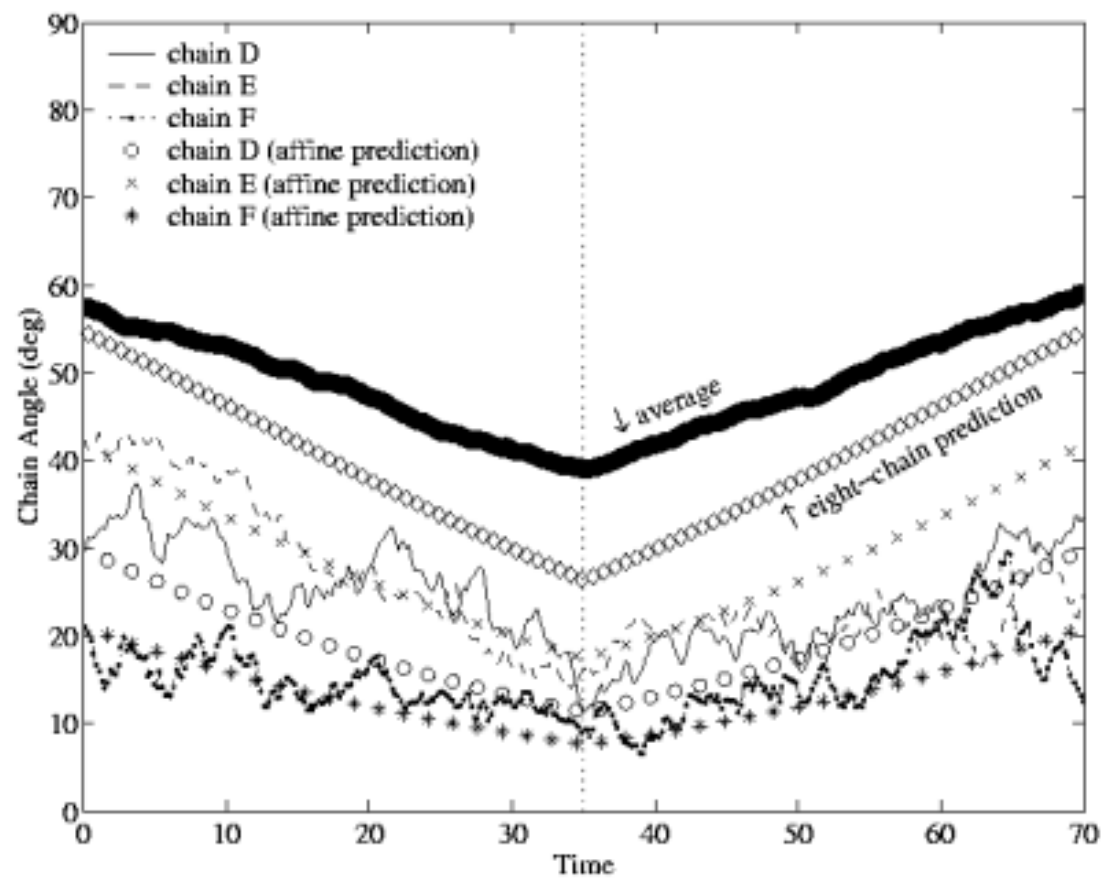

Figure 13: Chain angles as a function of simulation time.

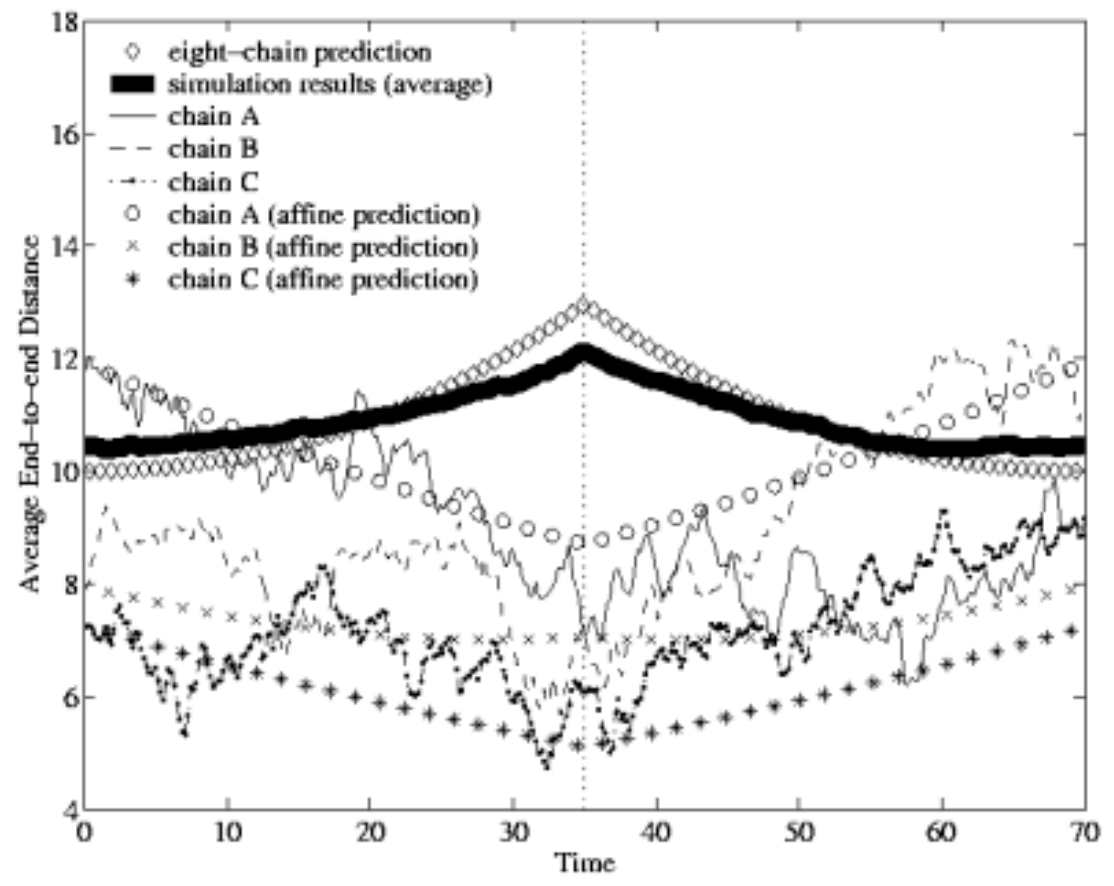

Figure 14: Chain end-to-end distance as a function of simulation time. 


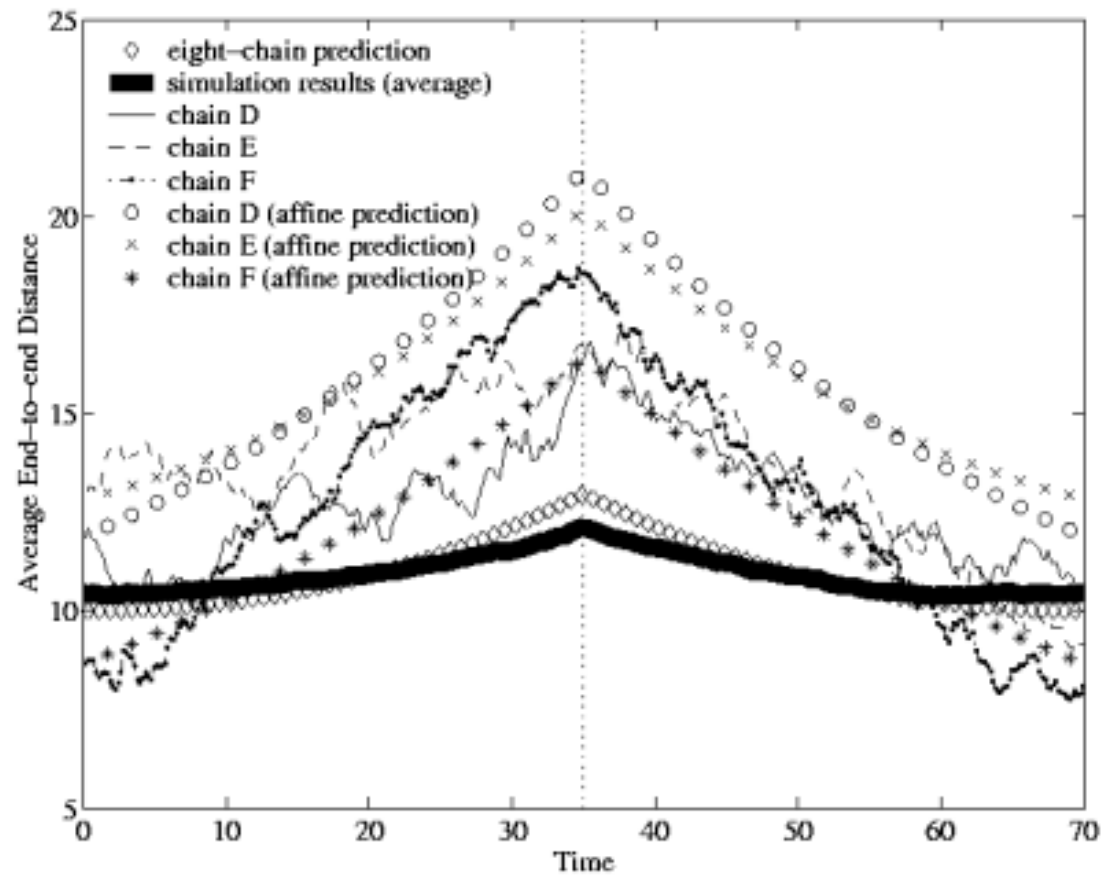

Figure 15: Chain end-to-end distance as a function of simulation time.

deformation, the stretch of the representative chains is given by

$$
\lambda_{\text {chain }}=\sqrt{\frac{\lambda^{2}+2 / \lambda}{3}}
$$

and the end-to-end distance is thus:

$$
l=\lambda_{\text {chain }} l_{0} .
$$

The chain angle is

$$
\beta=\operatorname{acos}\left[\frac{\lambda}{\sqrt{\lambda^{2}+2 / \lambda}}\right] .
$$

Figures 12 to 15 show the individual chains to basically deform in an affine manner. While the chain end-to-end lengths and angles do not evolve in a monotonic manner in the MD simulations, the trend is to follow the affine deformation result overall for these moderate stretches. The MD computed average chain end-to-end length and angle are found to compare favorably with the 8-chain model predictions where the averaged chain is stretching and rotating toward the principal stretch direction similar to that predicted in the 8-chain model. These comparisons would be interesting to pursue into the nonGaussian larger stretch region to observe the breakdown in affine deformation of all chains and the apparent robustness of the 8-chain effective network representation in this regime.

So far the discussion has been limited to topological properties of the molecular network, but from the simulation it is possible to study a number of other quantities as well. The variation in the different energy quantities as a function of simulation time is shown in Figure 16 illustrating that the kinetic, bonded, and non-bonded energies all stay virtually constant during the simulation. This observation is in agreement with traditional rubber elasticity theory in which the deformation resistance is entropy based. 


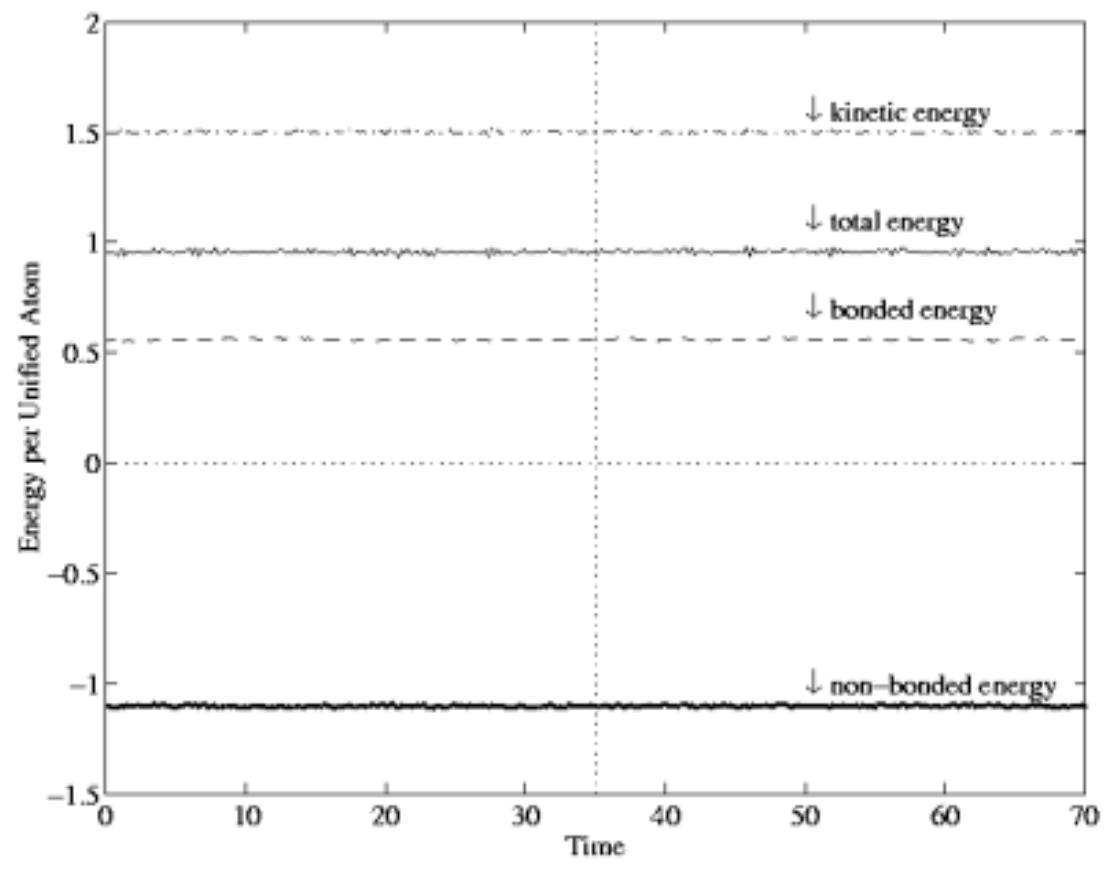

Figure 16: Energies of the RVE as a function of simulation time.

The variation in the pressure on the lateral sides of the RVE as a function of simulation time is shown in Figure 17. Since the MD simulation is run at constant volume, the pressure varies with deformation state, but as shown in the figure, the change in lateral pressure is relatively small. Simulations comparing $N V \theta$ with $N V P$ show nearly identical results [Bergström, 1999] in terms of structure and stress-strain behavior.

Figure 18 shows the simulated stress-strain response illustrating the same qualitative features that are observed in traditional experimental mechanical tests: the tangent modulus is initially a decreasing function of strain but soon becomes relatively constant (as long as the strain is not too large such that the limited chain stretch is approached), and at the strain reversal there is a significant drop in stress causing a hysteretic energy loss. Note that the relatively stiff initial response as well as the initial stiff unloading response correlate with a rapid change in bond angle shown earlier in Figure 11. Also in the figure is shown the prediction from Gaussian rubber elasticity theory: $\sigma=N k_{B} \theta\left(\lambda^{2}-1 / \lambda\right)$, which when normalized following the procedure discussed in Section 3 can be written $\tilde{\sigma}=\tilde{N}\left(\lambda^{2}-1 / \lambda\right)$ where $\tilde{N}$ is the number of effective chains in the RVE divided by the volume of the RVE. It is important to realize that due to entanglement effects the number of effective chains is not the same as the number of real chains, it will be much larger. The effective chain length is here taken to be $n=5 b$, the motivation for using this small value is that no bond-torsional or bond-angle potentials were used hence giving the chains very large flexibility. It is also an indication of the unified atom principle, each particle in the simulation represents a monomer or a group of monomers, and not individual atoms. As will be shown in Section 6, an effective chain length of $n=5 b$ also gives very good agreement with the simulated stress-strain response in uniaxial compression. In Langevin chain statistics the locking stretch of a chain is given by the square-root of the number of effective bonds in the chain, which when using the simulation results is predicted to be $\lambda_{\text {chain }}=2.236$. By using the 8-chain model this can be correlated to macroscopic deformations: the locking stretch in uniaxial tension becomes 3.84 and the locking stretch in uniaxial compression becomes 0.134 , both of these are reasonable values that would 


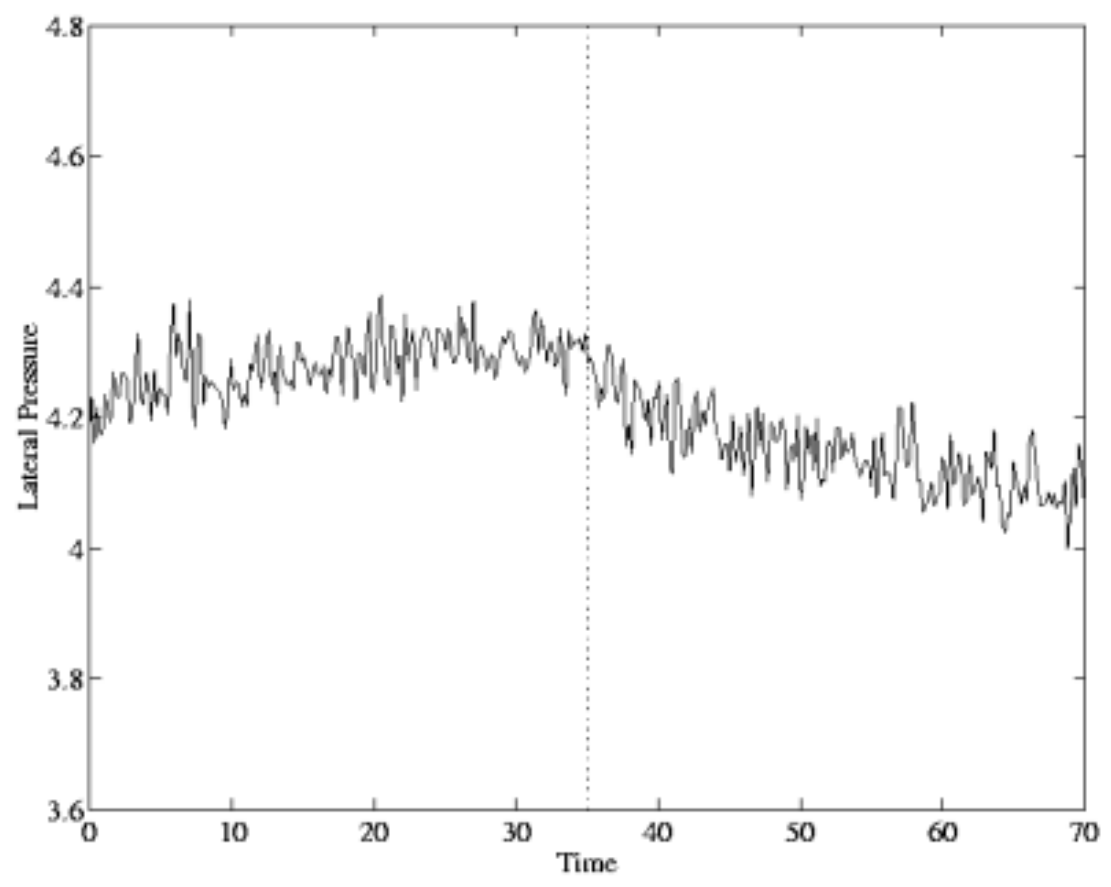

Figure 17: Lateral pressure $\left(T_{y y}+T_{z z}\right) / 2$ in the RVE as a function of simulation time.

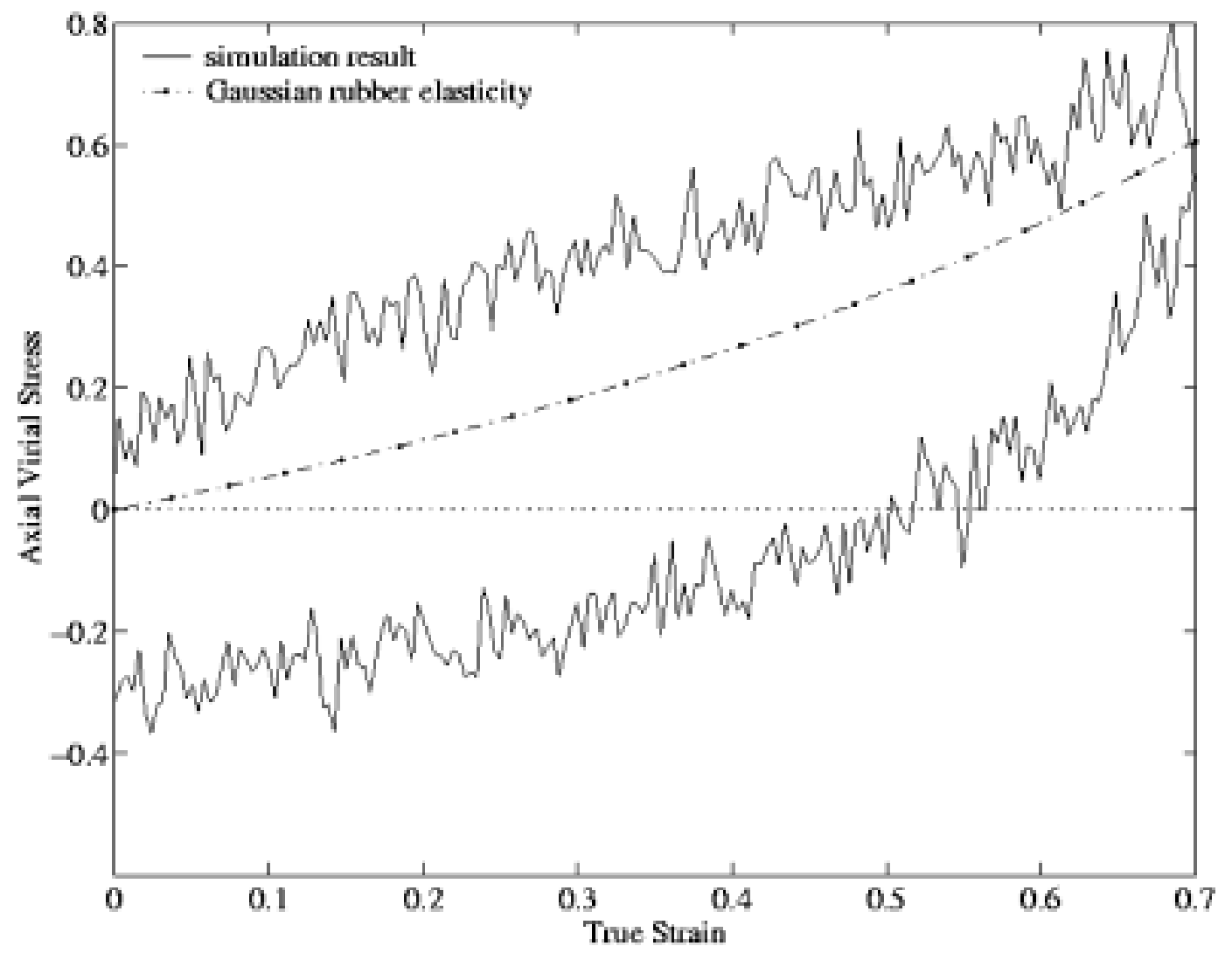

Figure 18: Stress-strain response of the RVE. 
be interesting to examine in more detail in another investigation.

Since the energy of the system consists of two parts: bonded and non-bonded contributions, it is possible to decompose the total stress into contributions from those two interactions. Results from this decomposition are shown in Figure 19. The unmodified axial stress $\left(T_{x x}\right)$ is found to contain equal contributions from the bonded and non-bonded sources where the bonded contribution originate from the bonds rotating toward the principal stretch direction. The lateral stresses arise from non-bonded contributions due to the constant volume constraint. Note that in this figure all stress components have been vertically shifted so that they start from zero. This procedure allows for a more direct comparison between the changes in magnitudes of the different stress components.

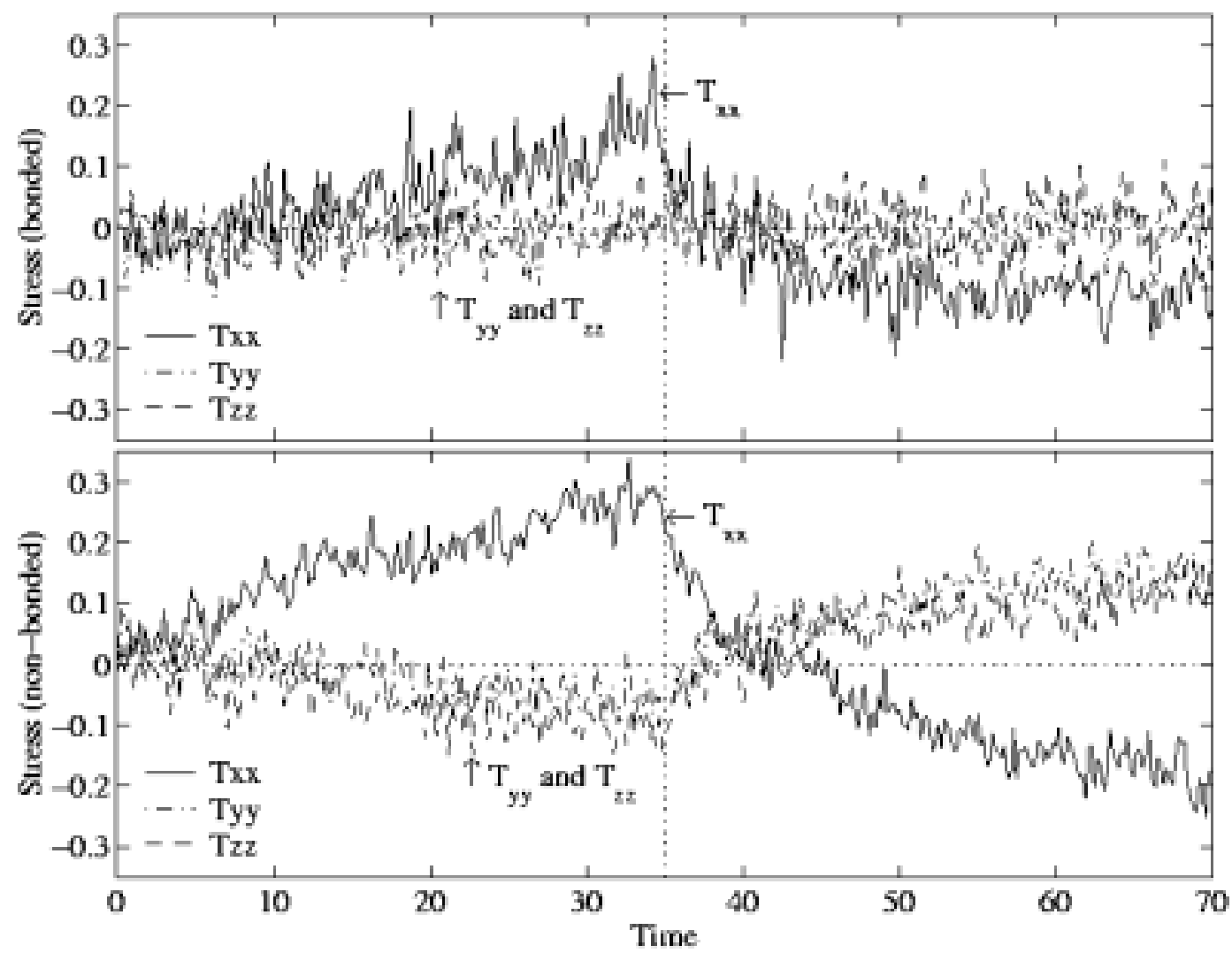

Figure 19: Decomposition of the stress in to bonded and non-bonded contributions. 


\section{Compression Results}

A representative volume element (RVE) containing 200 chains is now deformed in compression to a final strain of -0.7 . To allow for a direct comparison between the tension and compression simulations the same deformation rate was chosen as in the previous section, see Figure 20. The distribution of the bond angles in the simulation cell is presented in the bond angle pole figures shown in Figure 20. From the pole figures it is clear that during the compression segment the average bond angle increases since on average the bonds rotate away from the loading axis. This is further demonstrated in Figure 21 where the average bond angle is shown to be a strong function of the applied deformation. As in the tension case the average bond angle only undergoes a relatively small change during the deformation, in this case the average angle increases about $5^{\circ}$ when the RVE is compressed to a true strain of -0.7 . Also similar to the tension case is the rapid change in bond angles at the strain reversals and the overshoot in bond angles at the end of the deformation cycle.

Figures 22 and 23 depict the evolution in chain angle with deformation for six randomly selected chains as well as the corresponding evolution evolution in the average chain angle; similarly, Figures 24 and 25 depict the evolution in chain end-to-end length for the same six chains as well as the averaged (over all 200 chains) behavior. Chains initially nearly orthogonal to the loading axis (chains $a, b$ ) are found to remain orthogonal and stretch with the deformation; a chain initially at $70^{\circ}$ is found to rotate away from the loading axes toward the principal stretch direction and to stretch. Chains initially oriented at angles less than $45^{\circ}$ to the loading axis are observed to rotate away from the loading axis toward the maximum principal stretch direction and their lengths are found to remain relatively constant or to even decrease with the applied strain. While the individual chains exhibit the basic trend just discussed, we note that the behavior is not monotonic but exhibit sudden increases/decreases in chain angle and length as deformation is monotonically increased. The averaged strain behavior is found to monotonically stretch with applied strain and to monotonically rotate towards the maximum principal stretch direction away from the loading direction.

Figures 22 to 25 also depict the individual chain behaviors predicted assuming affine deformation of all chains; the 8-chain model is used to predict the averaged or effective chain behavior. The individual chains are found to basically follow the affine deformation assumption. The averaged chain behavior is found to basically follow the affine deformations assumption. The averaged chain behavior is found to be in excellent agreement with the 8-chain model predictions, suggesting that the 8-chain model does capture an effective network response and this appears to be the reason for its success (again, this would be interesting to probe into the large strain regime of behavior).

The variation in lateral pressure (Figure 26) is similar to the tension case. In compression, the lateral pressure decreases a small amount; whereas, in tension the lateral pressure had increased a small amount with strain (in both cases about 2.5\%). These changes in lateral pressure, although small, indicates that the simulated material is not incompressible.

The compressive stress-strain behavior is shown in Figure 27 and decomposed into bonded and non-bonded contributions in Figure 28. The stress-strain behavior is similar in character to that obtained earlier in tension. In this figure is also plotted the results from Gaussian rubber elasticity theory using an effective chain length of $n=5 b$, as was discussed for the tension case in Section 5 . The stress decomposition shows a strong bonded contribution to the lateral stresses since the bonds are rotating towards these directions whereas the unmodified axial stress $\left(T_{x x}\right)$ is dominated by non-bonded contributions. 


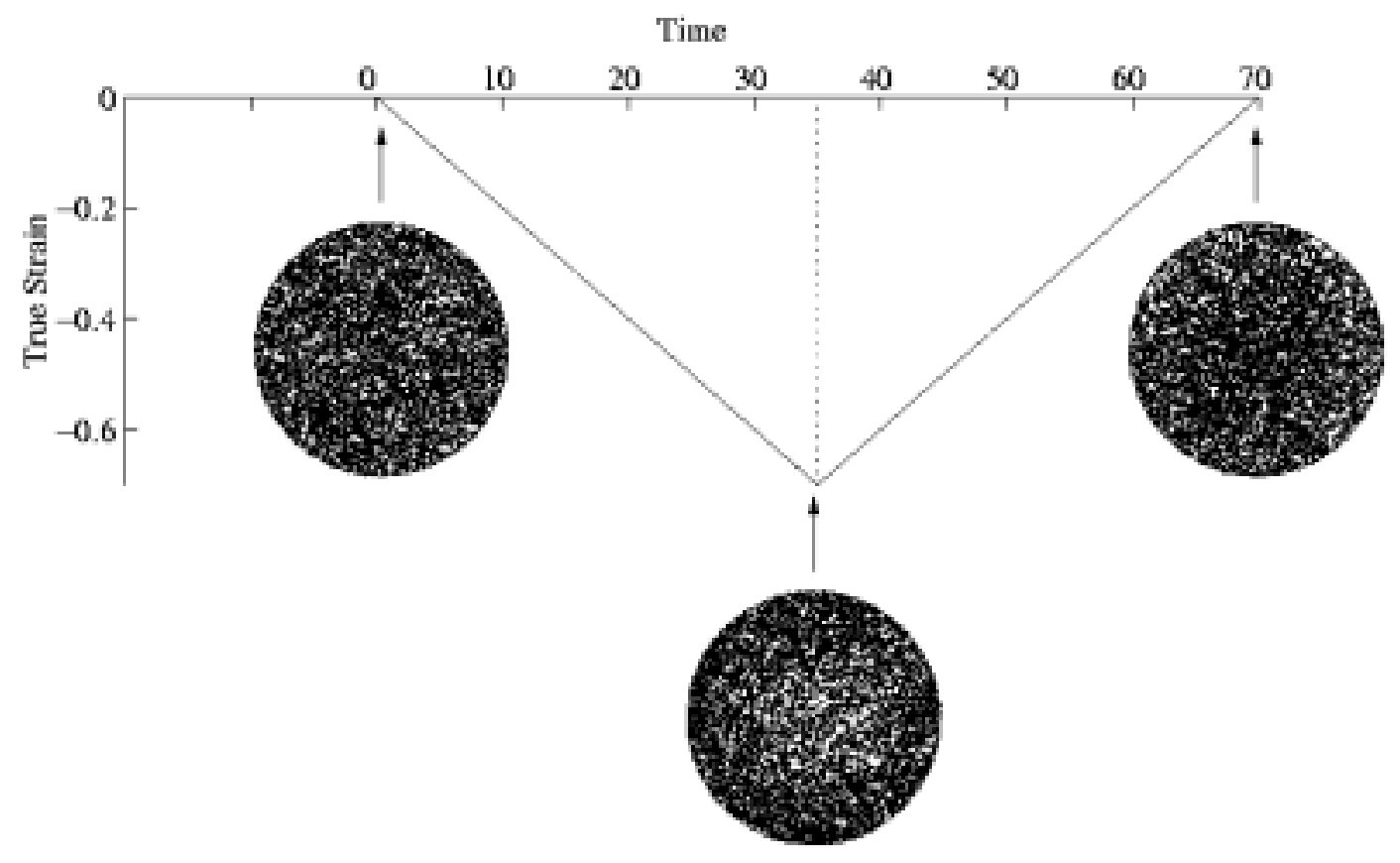

Figure 20: Applied strain history with bond angle pole figures.

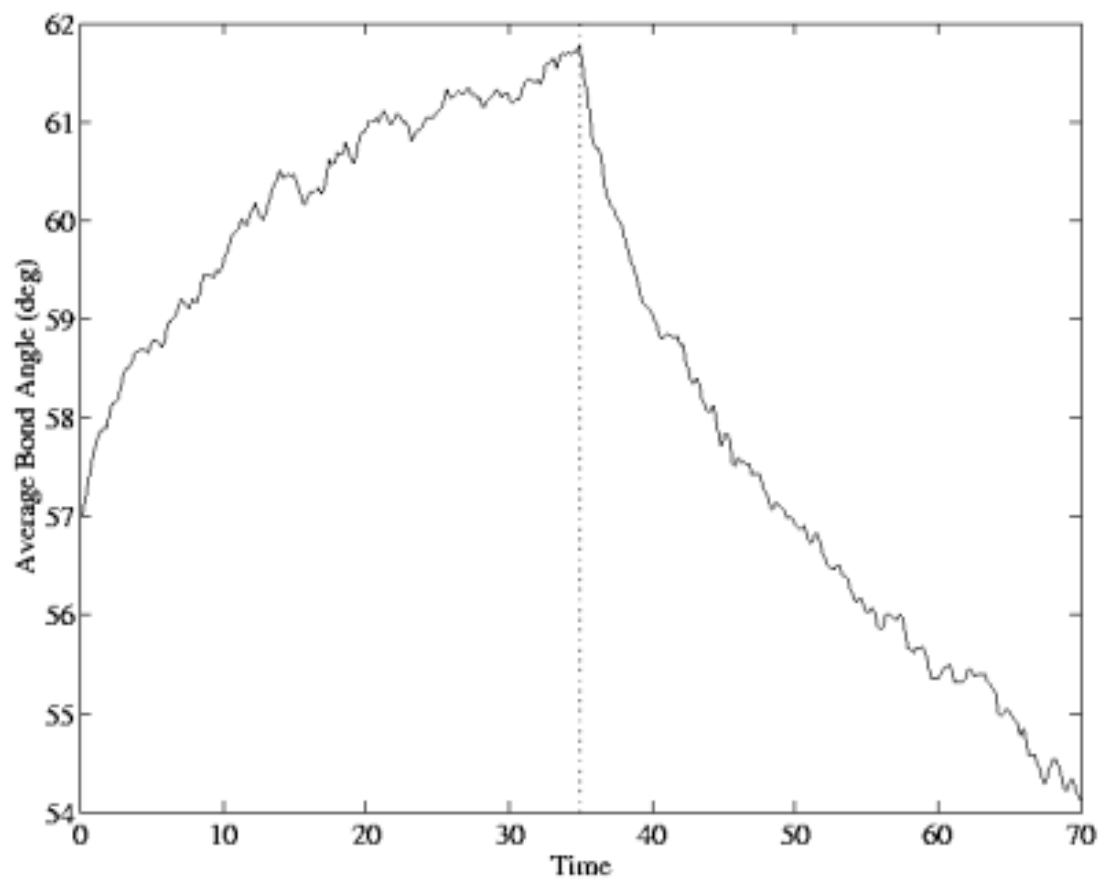

Figure 21: Average bond angle of as a function of simulation time. 


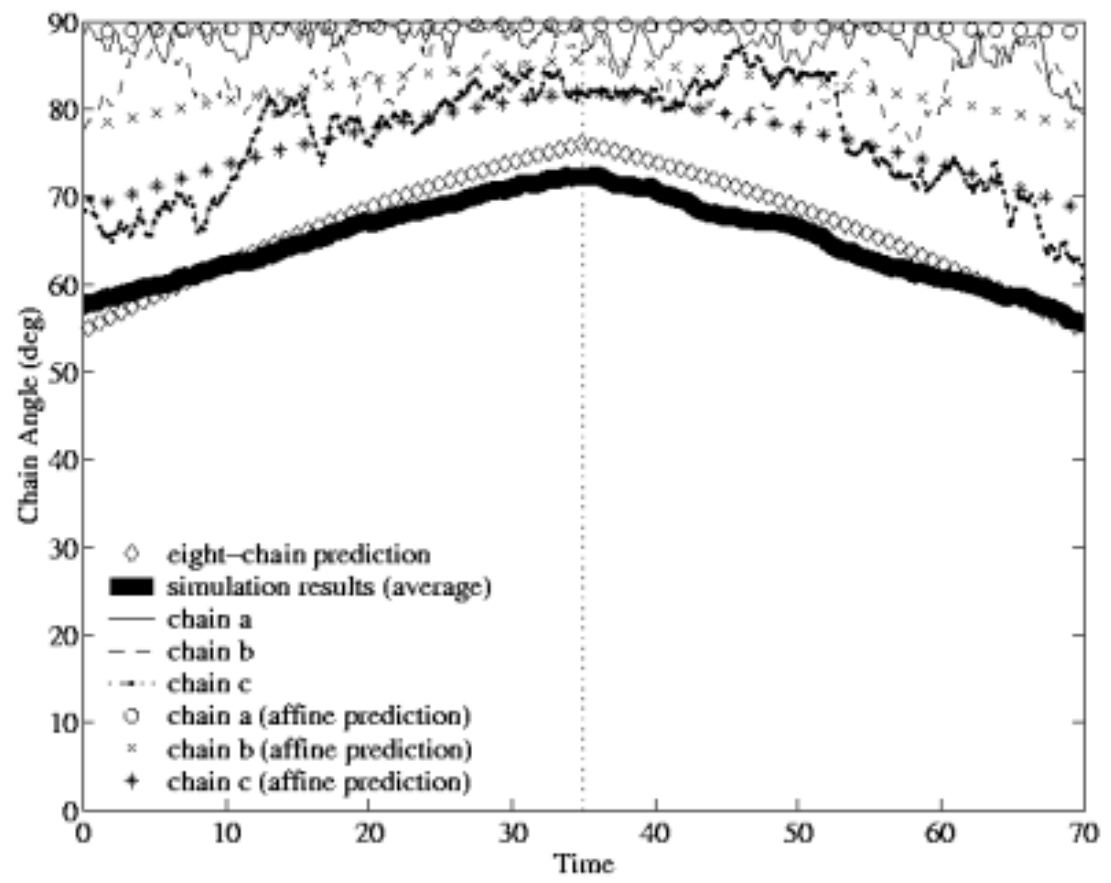

Figure 22: Average end-to-end angle for three chains.

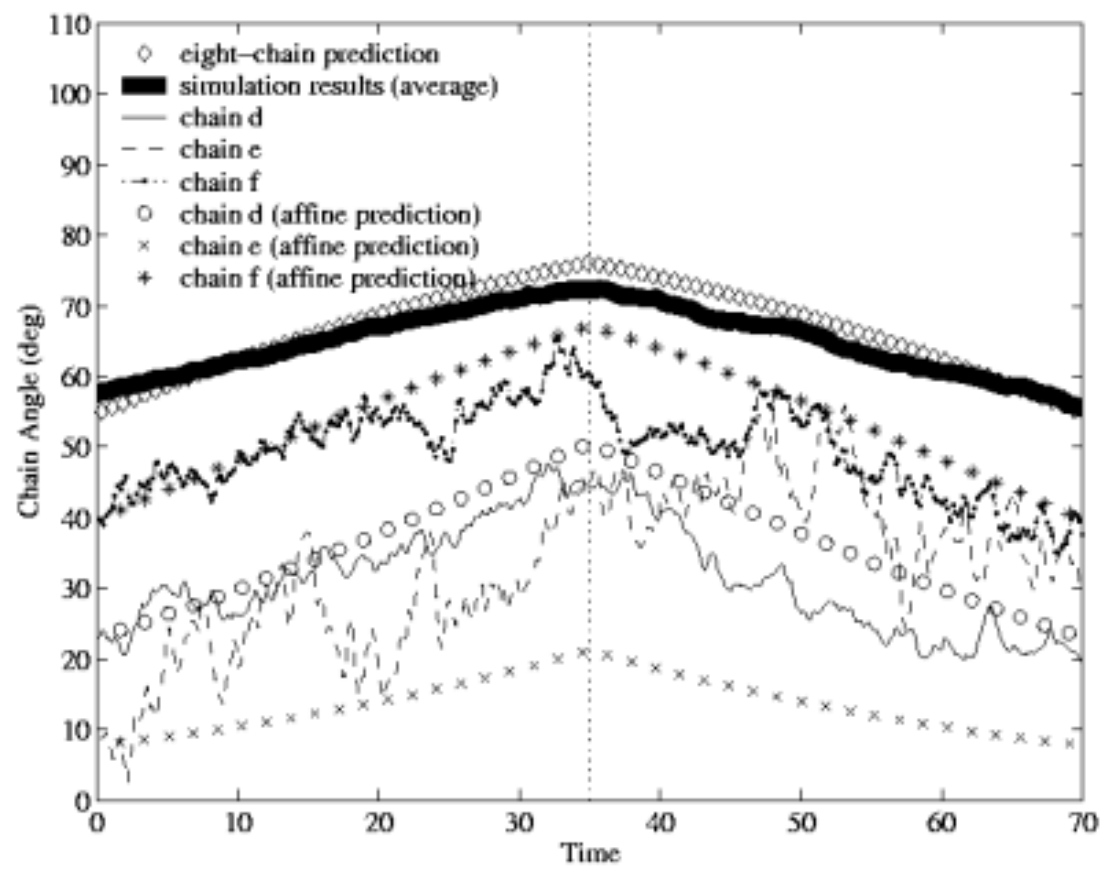

Figure 23: Average end-to-end angle for three chains. 


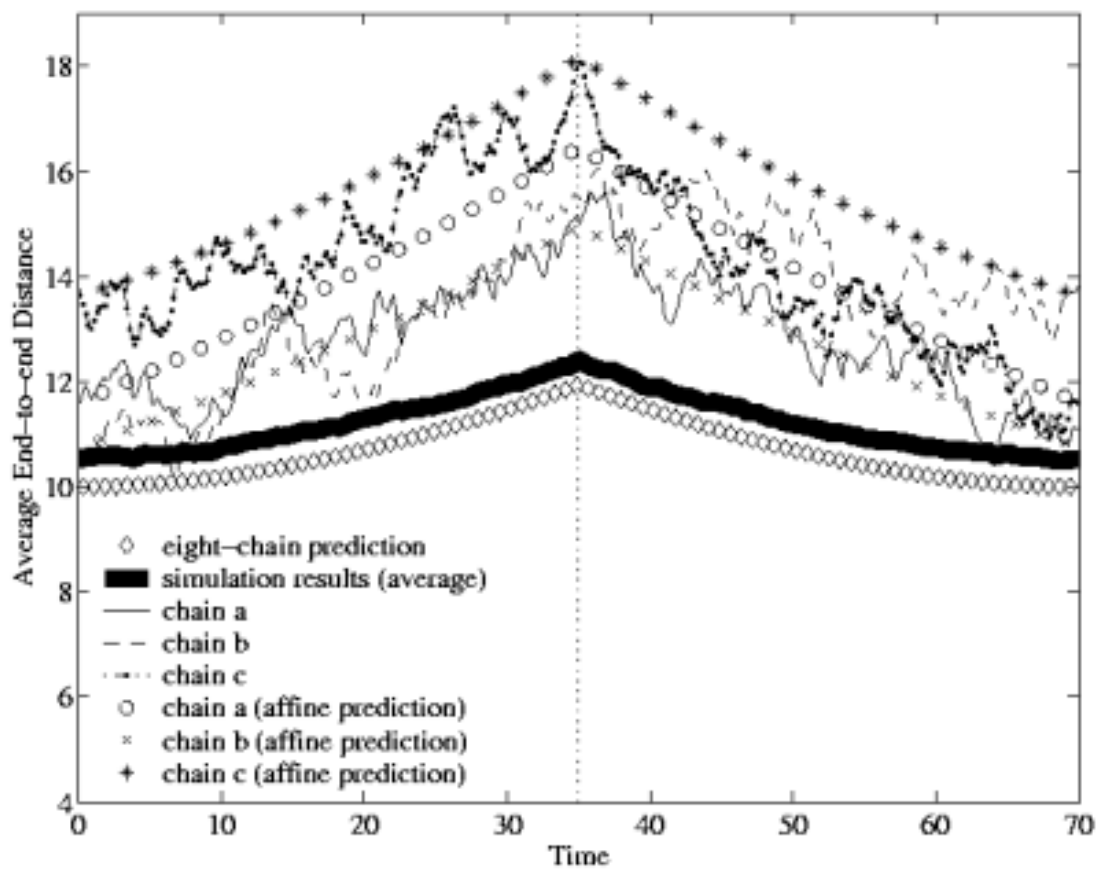

Figure 24: Average chain stretch for three chains.

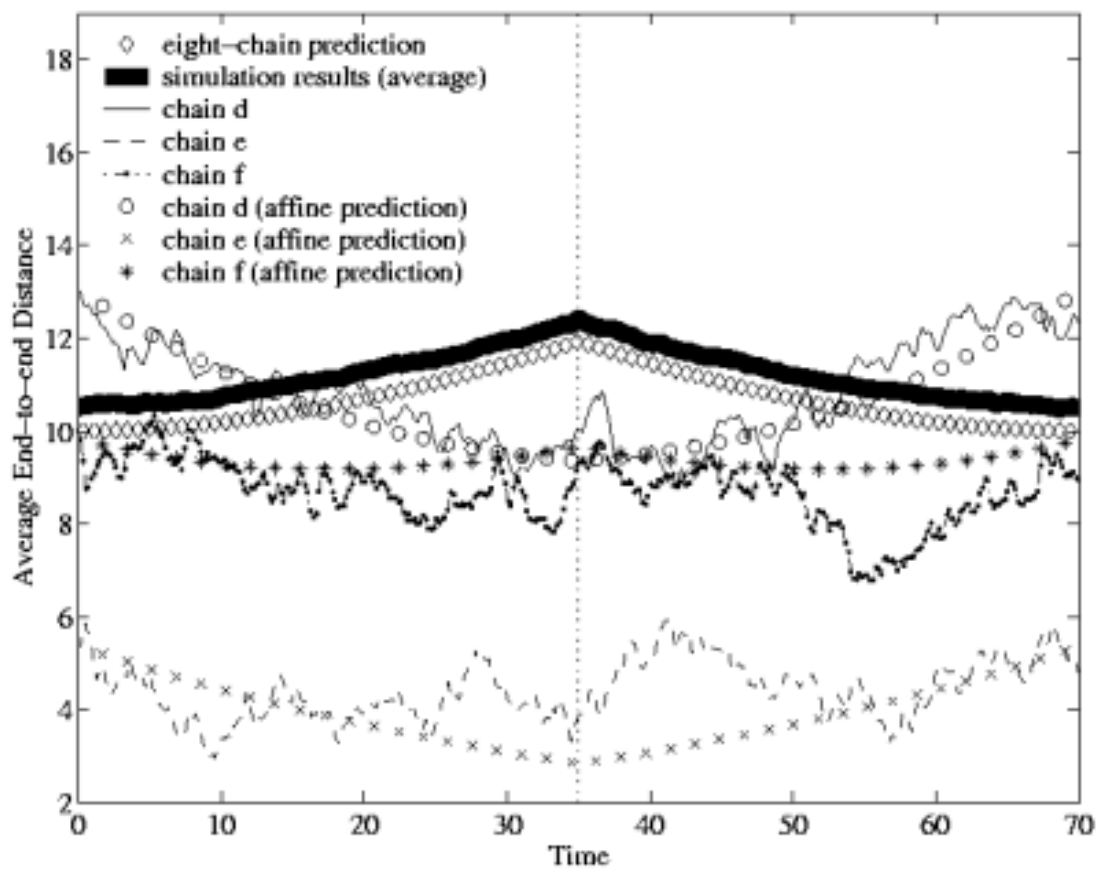

Figure 25: Average chain stretch for three chains. 


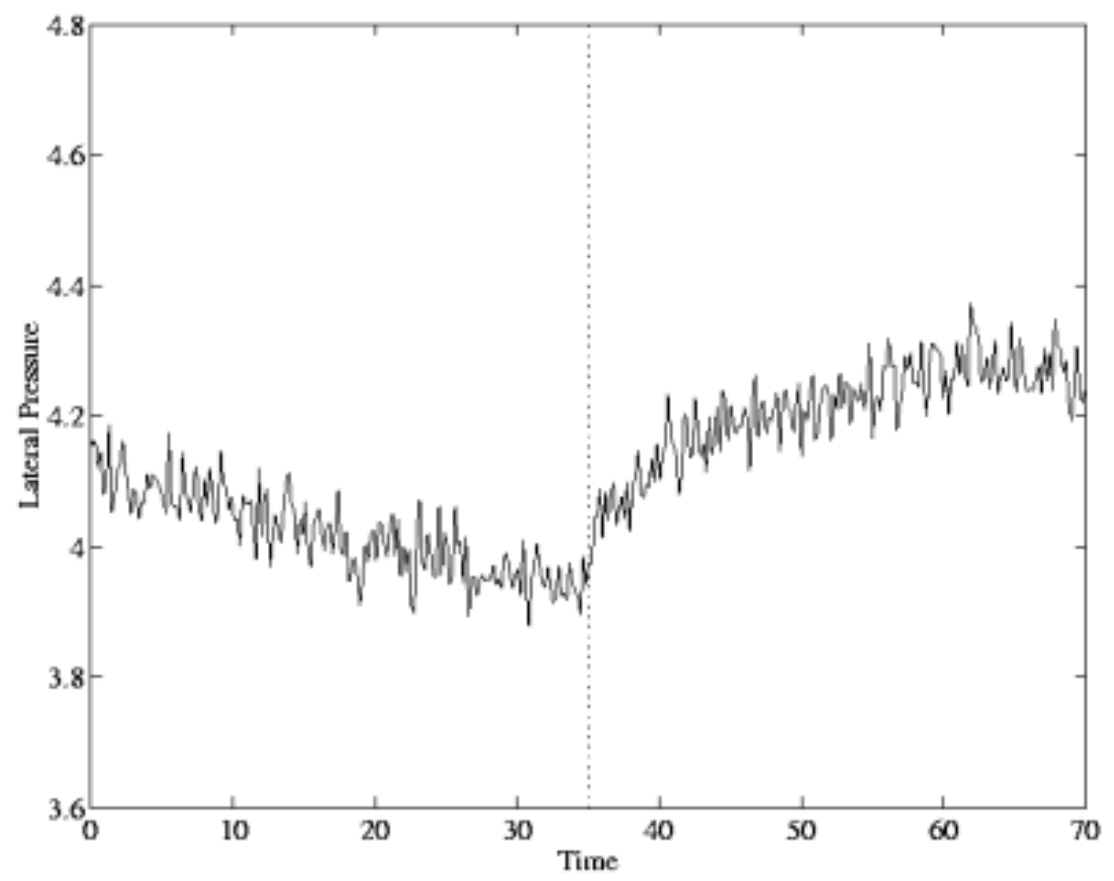

Figure 26: Lateral pressure in the RVE as a function of simulation time.

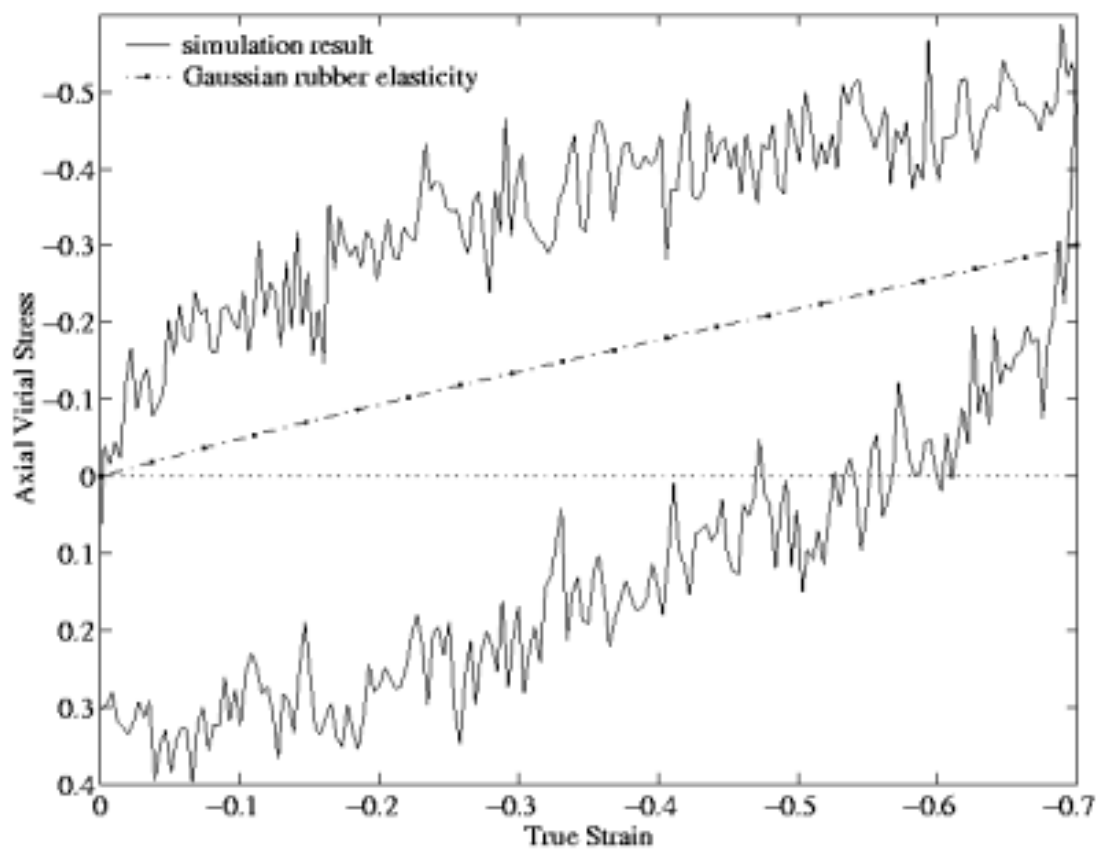

Figure 27: Stress-strain response of the RVE. 


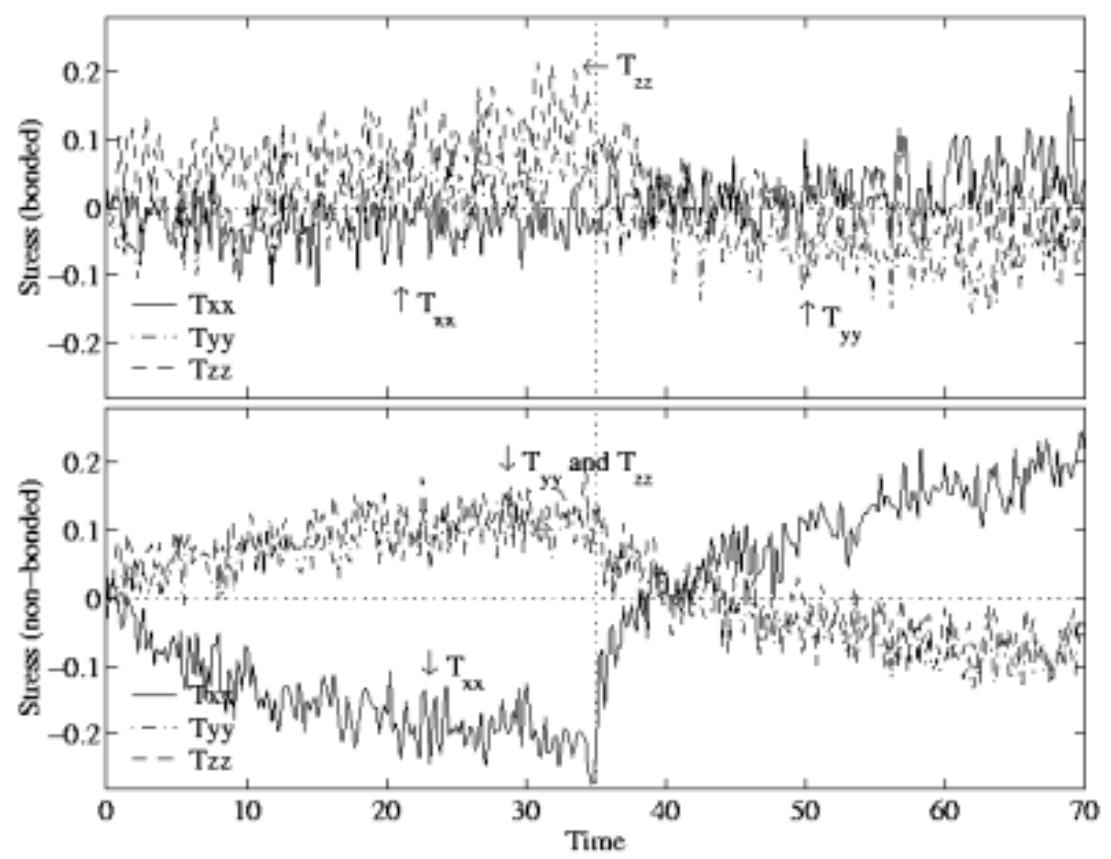

Figure 28: Decomposition of the stress in to bonded and non-bonded contributions. 


\section{Conclusions}

In addition to traditional experiments and theoretical modeling it is often helpful to use "computer experiments" to facilitate the understanding of physical processes. In polymer science these are often molecular dynamics (MD) or Monte Carlo (MC) simulations. In this work it has been demonstrated that molecular simulations can be a useful tool for studying the mechanical behavior of elastomeric networks. By comparing results from MD simulations in both compression and tension it has been shown that this type of simulation can produce results that are in good in qualitatively agreement with experimental data. A number of interesting observations are directly obtained from the simulations. For example, the simulations clearly demonstrate a very strong correlation between the average bond angle and the stress in the system. This observation does not seem to be very well documented in the literature, but can easily be understood in terms of the virial stress theorem. The change in average bond angle with applied deformation is further shown to be relatively small: applying a true strain of $+/-0.7$, for example, only causes a change in the average bond angle with about $-/+6^{\circ}$. Similarly the change in chain angle with applied deformation is also shown to be small. Here, applying a true strain $+/-0.7$ causes a change in chain angle of about $-/+18^{\circ}$. This observation is in good agreement with the prediction from the 8-chain model [Arruda and Boyce, 1993]. From the simulations it is also possible to follow the average chain stretch as a function of applied deformation. It is interesting to note that the average chain stretch only becomes about 1.2 when the applied stretch is 2 . This observation is also in good agreement with the 8-chain model. The simulations have also indicated that both in tension and compression on average the chains deform in an affine manner.

Since the energy of the system consists of two parts: bonded and non-bonded contributions, it is possible to decompose the total stress into contributions from those two interactions. Results from this decomposition indicate that the contribution from the bonded potential is mainly causing a change

in $T_{x x}$ with applied deformation, and that the contribution from the non-bonded potential causes a simultaneous increase in the axial stress and a reduction in the lateral stresses.

In conclusion, molecular simulations of the type used in this work appears to be an interesting compliment to traditional experiments when developing constitutive equations. It is particularly interesting to see how many features of the simulation results are universal, i.e.that do not depend on the particular techniques or parameters used in the simulations, and the very good qualitative agreement with experimental data. In a future study it would be interesting to extend the work presented here to include also a more careful investigation of different crosslinking densities and time-dependent effects as well as studying large deformations where the limited stretch of the chains is approached thus enabling a test of inverse Langevin statistics.

\section{Acknowledgement}

This research was funded, in part, by the US NSF through grant no. CMS-9622526 and through the NSF MRSEC MIT CMSE through grant no. DMR-98-08941.

\section{References}

M. P. Allen and D. J. Tildesley. Computer simulation of liquids. Oxford University Press, 1987. 6 
E. M. Arruda and M. C. Boyce. A three-dimensional constitutive model for the large stretch behavior of rubber elastic materials. J. Mech. Phys. Solids., 41(2):389-412, 1993. 3, 27

J. S. Bergström. Large Strain Time-Dependent Behavior of Elastomeric Materials. PhD thesis, MIT, 1999. 18

C. Chui and M. C. Boyce. Monte Carlo modeling of amorphous polymer deformation: Evolution of stress with strain. Macromolecules, 32:3795-3808, 1999. 4

E. R. Duering. Structure and relaxation of end-linked polymer networks. J. Chem. Phys., 101(1): 8169-8192, 1994. 5

J. Gao and J. Weiner. Macromolecules, 20:2520, 1987. 5

R. W. Hockney, S. P. Goel, and J. W. Eastwood. High-resolution computer models of a plasma. J. Comput. Phys., 14:48, 1974. 4

W. G. Hoover. Canonical dynamics: Equilibrium phase-space distributions. Phys. Rev. A, 31(3): 1695-1697, 1985. 6

W. Kuhn and F. Grün. Kolloid Z., 101:248, 1942. 2

S. Nosé. A molecular dynamics method for simulation in the canonical ensemble. Molecular Physics, $52(2): 255-268,1984.6$

L. R. Treloar. The Physics of Rubber Elasticity. Oxford University Press, 1975. 2

L. R. G. Treloar. The elasticity of a network of long-chain molecules-ii. Trans. Faraday Soc., 39: 241-246, 1943. 2

L. R. G. Treloar. Trans. Faraday Soc., 50:881, 1954. 3

L. Verlet. Computer experiments on classical fluids. i. thermodynamic properties of lennard-jones molecules. Phys. Rev., 159:98, 1967. 4

F. T. Wall. J. Chem. Physics, 10:485, 1942. 2

M. C. Wang and E. J. Guth. J. Chem. Phys., 20:1144, 1952. 3

P. D. Wu and E. van der Giessen. On improved network models for rubber elasticity. J. Mech. Phys. Solids, 41(3):427-456, 1993. 3 